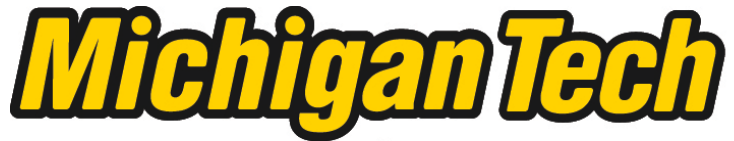 \\ Michigan Technological University Create the Future Digital Commons @ Michigan Tech
}

Dissertations, Master's Theses and Master's Reports - Open

Dissertations, Master's Theses and Master's

Reports

2014

\section{A SURVEY OF DISTANCE MAGIC GRAPHS}

Rachel Rupnow

Michigan Technological University

Follow this and additional works at: https://digitalcommons.mtu.edu/etds

Part of the Mathematics Commons

Copyright 2014 Rachel Rupnow

\section{Recommended Citation}

Rupnow, Rachel, "A SURVEY OF DISTANCE MAGIC GRAPHS", Master's report, Michigan Technological University, 2014.

https://doi.org/10.37099/mtu.dc.etds/829

Follow this and additional works at: https://digitalcommons.mtu.edu/etds

Part of the Mathematics Commons 


\title{
A SURVEY OF DISTANCE MAGIC GRAPHS
}

\author{
By
}

Rachel Rupnow

\begin{abstract}
A REPORT
Submitted in partial fulfillment of the requirements for the degree of MASTER OF SCIENCE

In Mathematical Sciences
\end{abstract}

MICHIGAN TECHNOLOGICAL UNIVERSITY

2014

(C)2014 Rachel Rupnow 

This report has been approved in partial fulfillment of the requirements for the Degree of MASTER OF SCIENCE in Mathematical Sciences.

Department of Mathematical Sciences

Report Advisor: Donald Kreher

Committee Member: Melissa Keranen

Committee Member: Scott Miers

Department Chair: Mark Gockenbach 



\section{Contents}

1 Introduction $\quad 9$

2 Distance Magic Graphs 13

2.1 Basic Results . . . . . . . . . . . . . . . . . . . . . . 13

2.2 Regular Graphs . . . . . . . . . . . . . . . . . . . . . 16

2.3 Multipartite Graphs . . . . . . . . . . . . . . . . . . . . . . . . . . . 19

2.4 Graph Products . . . . . . . . . . . . . . . . . . . . . 22

2.4.1 Lexicographic Product . . . . . . . . . . . . . . . . 22

2.4.2 Direct Product . . . . . . . . . . . . . . . . . . 24

2.4.3 Cartesian Product . . . . . . . . . . . . . . . 25

2.4.4 Strong Product . . . . . . . . . . . . . . . . . 26

2.5 Join Graphs . . . . . . . . . . . . . . . . . . . . . . . . . . . . . . . . . . . . . . . . . . . . . . . .

2.6 Splitting Graphs . . . . . . . . . . . . . . . . . . . . . 29

2.7 Embedding Theorems . . . . . . . . . . . . . . . . . . . . . . . . . . . . . . . . . .

2.8 Matrix Representation . . . . . . . . . . . . . . . . . . 30

2.9 Lifted Magic Rectangles and $p$-regular graphs . . . . . . . . . . . . . 31

2.10 Distance Magic Constants . . . . . . . . . . . . . . . . . . 31

3 Related Graph Labelings 33

3.1 Balanced Distance Magic Graphs . . . . . . . . . . . . . . . 33

3.2 Other Distances . . . . . . . . . . . . . . . . . . . . . 33

3.2.1 Closed Distance Magic Graphs . . . . . . . . . . . . . . . . . 34

3.2.2 Distances Greater than 1 . . . . . . . . . . . . . . . 37

3.3 Distance Antimagic Graphs . . . . . . . . . . . . . . . . . 41

4 Different Labels $\quad 45$

4.1 Group Distance Magic Graphs . . . . . . . . . . . . . . . . . . . . . 45

$4.1 .1 \mathbb{Z}_{n}$-Distance Magic Graphs . . . . . . . . . . . . . . . . . 45

4.1.2 General $\Gamma$-Distance Magic Graphs . . . . . . . . . . . . . . . . 47

4.2 Group Distance Antimagic Graphs . . . . . . . . . . . . . . . . 54

4.2.1 $\mathbb{Z}_{n}$-Distance Antimagic Graphs ............ 54

4.2.2 $\Gamma$-Distance Antimagic Graphs . . . . . . . . . . . . . 55 
5 Applications $\quad 61$

6 Open Problems $\quad 65$

$\begin{array}{ll}\text { Bibliography } & 71\end{array}$ 
Abstract
In this report, we survey results on distance magic graphs and some closely related graphs. A distance magic labeling of a graph $G$ with magic constant $k$ is a bijection $\ell$ from the vertex set to $\{1,2, \ldots, n\}$, such that for every vertex $x$

$$
\sum_{y \in N_{G}(x)} \ell(y)=k
$$

where $N_{G}(x)$ is the set of vertices of $G$ adjacent to $x$. If the graph $G$ has a distance magic labeling we say that $G$ is a distance magic graph.

In Chapter 1, we explore the background of distance magic graphs by introducing examples of magic squares, magic graphs, and distance magic graphs.

In Chapter 2, we begin by examining some basic results on distance magic graphs. We next look at results on different graph structures including regular graphs, multipartite graphs, graph products, join graphs, and splitting graphs. We conclude with other perspectives on distance magic graphs including embedding theorems, the matrix representation of distance magic graphs, lifted magic rectangles, and distance magic constants.

In Chapter 3, we study graph labelings that retain the same labels as distance magic labelings, but alter the definition in some other way. These labelings include balanced distance magic labelings, closed distance magic labelings, $D$-distance magic labelings, and distance antimagic labelings.

In Chapter 4, we examine results on neighborhood magic labelings, group distance magic labelings, and group distance antimagic labelings. These graph labelings change the label set, but are otherwise similar to distance magic graphs.

In Chapter 5, we examine some applications of distance magic and distance antimagic labeling to the fair scheduling of tournaments.

In Chapter 6, we conclude with some open problems. 


\section{Acknowledgements}

I want to extend special thanks to Don Kreher for his guidance and encouragement. I thank Sylwia Cichacz and Dalibor Froncek for the papers and assistance they provided. I would also like to thank the Mathematics Department at Michigan Technological University for their willingness to spend time explaining outside of class. Additionally, I want to thank my Mom and Dad for their love and support. Above all, I thank God for giving me the ability to learn and succeed. Soli Deo Gloria! 


\section{Chapter 1}

\section{Introduction}

One mathematical object that has delighted mathematicians and laypeople for generations is the magic square. A magic square is an array in which each entry is distinct, but each row, column, and diagonal adds to the same number. Perhaps the most famous example is the $3 \times 3$ magic square below with constant sum 15 .

\begin{tabular}{|l|l|l|}
\hline 8 & 1 & 6 \\
\hline 3 & 5 & 7 \\
\hline 4 & 9 & 2 \\
\hline
\end{tabular}

Figure 1: $3 \times 3$ Magic Square

The first recorded magic square is in a Chinese manuscript dated around 2200 BC. Magic squares continued to appear in books and art in many cultures, including the Mayans, Hasua people of Africa, and Renaissance Europe. Even Benjamin Franklin created similar arrays with beautiful symmetries [45].

Because of this historical interest in magic squares, in 1963, Jiří Sedláček introduced magic labelings of graphs. A graph is an ordered pair $(V, E)$, consisting of a set $V$ of vertices and a set $E$, disjoint from $V$, of edges, together with an incidence function $\psi$ that associates with each edge of $G$ an unordered pair of (not necessarily distinct) vertices of $G$. [11] Sedláček defined a magic labeling of a graph $G=G(V, E)$ as a bijection $f$ from the edge set $E$ to a set of positive integers such that

- $f\left(e_{i}\right) \neq f\left(e_{j}\right)$ for all distinct $e_{i}, e_{j} \in E$, and

- $\sum_{e \in N_{E}(x)} f(e)$ is the same for every $x \in V$, where $N_{E}(x)$ is the set of edges incident to $x$. [50]

Like magic squares, magic labelings require a constant sum. Indeed, a magic square of side $n$ provides a magic labeling of the complete bipartite graph $K_{n, n}$. Let $\left\{r_{1}, r_{2}, \ldots, r_{n}\right\}$ and $\left\{c_{1}, c_{2}, \ldots, c_{n}\right\}$ be the bipartition of vertices. Then given $n \times n$ magic square $M$, define the magic labeling of $K_{n, n}$ by taking $f\left(r_{i}, c_{j}\right)$ to be the entry 


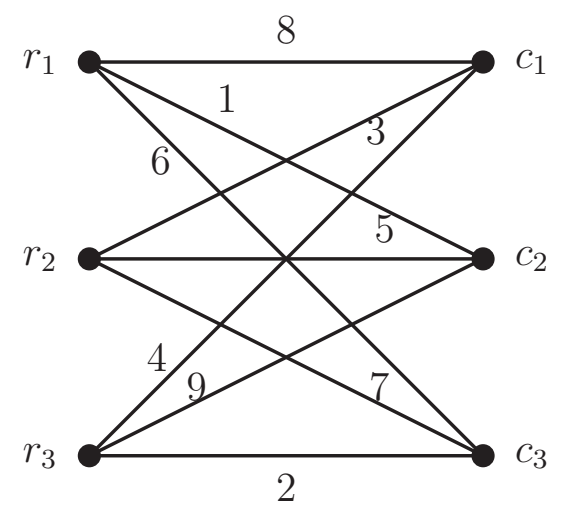

Figure 1.1: Magic Labeling of $K_{3,3}$ from the $3 \times 3$ Magic Square in Figure 1

in row $i$ and column $j$. Specifically, the magic square in Figure 1 provides a magic labeling of $K_{3,3}$ in Figure 1.1.

Another example, the graph in Figure 1.2, is a magic graph with magic constant 50. Notice this graph is not regular; that is, not every vertex is adjacent to the same number of vertices. Nevertheless, the constant sum property is retained.

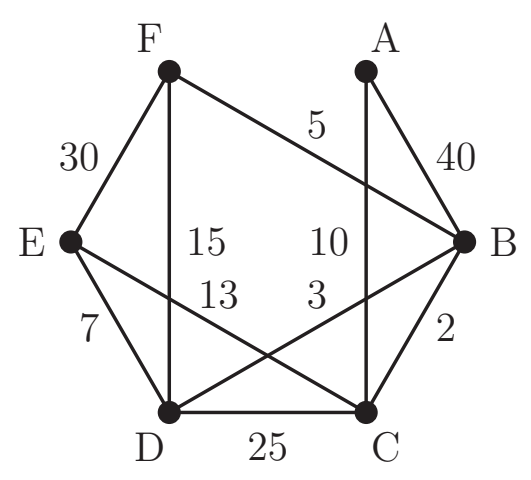

Figure 1.2: A Magic Graph

These magic labelings were easily extended to vertex-magic total labelings, edgemagic total labelings, and other magic labelings by many authors. For more information on magic graphs, see Gallian's survey. [28]

In general, graph labelings are often created in such a way as to provide information about the graph. For example, the distance between two vertices of graph $G$ is the minimum number of edges required to travel from one vertex to the other. This property is displayed in the following example. To address the problem of assigning radio frequencies, Griggs and Yeh defined the $L_{d}(2,1)$-labeling in which vertices of distance 1 and 2 must differ by $2 d$ and $d$ respectively [31]. Additionally, Gavoille, et 
al. created a method of labeling that allows one to compute the distance between vertices without any information about the graph except the labels [29].

This survey focuses on distance magic labelings, which combine the qualities of magic and distance labelings. A distance magic labeling of $G=G(V, E)$ is defined as a bijection $\ell: V \rightarrow\{1,2, \ldots, n\}$ with the property that there is a positive integer $k$, the magic constant, such that for every $x \in V(G), w(x)=\sum_{y \in N_{G}(x)} \ell(y)=k$, where $N_{G}(x)=\{y \in V(G): y$ is adjacent to $x\}$ is the open neighborhood of $x$. We call $w(x)$ the weight of vertex $x$ [40], [55]. Any graph that permits a distance magic labeling is a distance magic graph. Notice that unlike the original magic labelings, distance magic labelings are vertex, not edge labelings.

For example, the graph in Figure 1.3 is a distance magic graph with magic constant $k=14$.

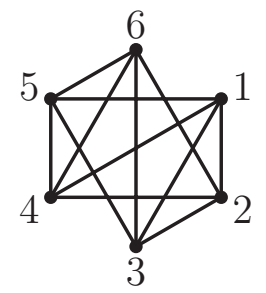

Figure 1.3: A Distance Magic graph

Because of the number of graph related papers and the difficulty in obtaining some papers, distance magic labelings have also been called 1-vertex-magic vertex labelings [40] and sigma labelings (or $\Sigma$-labelings) [9]. Each name emphasizes a different aspect of the labeling. "Distance magic" emphasizes the connection to magic squares and magic labelings. "1-vertex-magic" emphasizes the distance involved, which in the case of the paper that introduced the term, was a distance of 1 [40]. " $\Sigma$-labeling" emphasizes that the weight of each vertex is a constant sum. For clarity, we will use the term "distance magic" in this paper.

Distance magic labelings of a variety of graphs have been studied. Regular graphs, graph products, and multipartite graphs all admit distance magic labelings in circumstances we will explore. In addition, both distance magic labeling and the related topic of antimagic labeling can be directly applied to the fair scheduling of tournaments. 


\begin{tabular}{|c|c|}
\hline Symbol & Meaning \\
\hline \hline$V(G)$ & Vertices in graph $G$ \\
\hline$E(G)$ & Edges in graph $G$ \\
\hline$N_{G}(x)$ & Deighborhood of vertex $x$ in $G$ \\
\hline$d e g(x)$ & Degree of vertex $x$ \\
\hline$|G|$ & Minimum degree of the vertices of $G$ \\
\hline$\delta(G)$ & Maximum degree of the vertices of $G$ \\
\hline$\Delta(G)$ & Complete graph on $n$ vertices \\
\hline$K_{n}$ & Cyath on $n$ vertices \\
\hline$K_{m, n}$ & Complete bipartite graph with partices \\
\hline$P_{n}$ & Complement of graph $G$ \\
\hline$C_{n}$ & Symmetric difference of sets $A$ and $B$ \\
\hline$W_{n}$ & Wheel formed by joining $C_{n-1}$ to a center vertex \\
\hline $\bar{G}$ & Direct product of graphs $G$ and $H$ \\
\hline$A \triangle B$ & Cartesian product of graphs $G$ and $H$ \\
\hline$G \circ H$ & Strong product of graphs $G$ and $H$ \\
\hline$G \times H$ & Bowtie product of graphs $G$ and $H$ \\
\hline$G \square H$ & Join graph of graphs $G$ and $H$ \\
\hline$G \otimes H$ & Splitting graph of $G$ \\
\hline$G \bowtie H$ & Spectrum of $G$ \\
\hline$G+H$ & \\
\hline$S^{\prime}(G)$ & \\
\hline$S p(G)$ & \\
\hline
\end{tabular}




\section{Chapter 2}

\section{Distance Magic Graphs}

\subsection{Basic Results}

Many of these basic results were compiled in a previous Distance Magic Graphs survey [7]. We include proofs of some of these results to clarify the concepts.

The first two theorems provide necessary conditions and attributes for distance magic graphs.

The degree of vertex $x$, denoted $\operatorname{deg}(x)$, is the number of vertices adjacent to $x$. The order of a graph is the number of vertices in a graph.

Theorem 2.1. [35], [40], [55] A necessary condition for the existence of a distance magic labeling $\ell$ of a graph $G$ of order $n$ is

$$
k n=\sum_{x \in V} \operatorname{deg}(x) \ell(x),
$$

where $k$ is the magic constant.

Proof. Certainly the sum of the weights on all vertices is $k n$. Conversely, for each vertex $x \in V$, this sum counts the label on $x$ exactly $\operatorname{deg}(x)$ times. Thus the equation holds.

Theorem 2.2. [35], [40], [47] [55] Let $G$ be an $r$-regular distance magic graph of order $n$. Then $k=\frac{r(n+1)}{2}$.

Proof. Because the graph is $r$-regular, $\operatorname{deg}(x)=r$ for all vertices $x$. Substituting into our necessary condition from Theorem 2.1 yields

$$
k n=\sum_{x \in V} r \ell(x)
$$


Thus dividing by $n$ we have:

$$
\begin{aligned}
k & =\frac{r}{n} \sum_{x \in V} \ell(x) \\
& =\frac{r(n+1)}{2} .
\end{aligned}
$$

The next four theorems reveal types of graphs that are not distance magic.

Theorem 2.3. [35], [40], [47], [55] No r-regular graph with $r$-odd can be a distance magic graph.

Proof. By Theorem 2.2, $k=\frac{r(n+1)}{2}$. Thus if $r$ is odd, $n$ must be odd. However, it is impossible to draw such a graph. (The number of vertices of odd degree in any graph must be even.) Thus no $r$-regular graph with $r$-odd can be a distance magic graph.

Theorem 2.4. [35], [55] Let $G$ be a graph of order $n$ which has two vertices of degree $n-1$. Then $G$ is not a distance magic graph.

Proof. Let $x_{1}$ and $x_{2}$ be distinct vertices of degree $n-1$ on graph $G$, and suppose $G$ is distance magic with labeling $\ell$ and magic constant $k$.

Then

$$
\begin{aligned}
k & =\sum_{i=1}^{n} \ell\left(x_{i}\right)-\ell\left(x_{1}\right) \\
& =\sum_{i=1}^{n} \ell\left(x_{i}\right)-\ell\left(x_{2}\right) \\
& \Longrightarrow \ell\left(x_{1}\right)=\ell\left(x_{2}\right) .
\end{aligned}
$$

By definition, the vertex labels on distance magic graphs are distinct so $G$ cannot be distance magic.

Lemma 2.5. [40] If $G$ contains two vertices $x_{i}$ and $x_{j}$ such that $\left|N_{G}\left(x_{i}\right) \cap N_{G}\left(x_{j}\right)\right|=$ $\operatorname{deg}\left(x_{i}\right)-1=\operatorname{deg}\left(x_{j}\right)-1$, then $G$ has no labeling.

A $t$-matching of $G$ is a collection of $t$ independent edges of $G$.

Theorem 2.6. [51] Let $\{e\}$ denote a single edge and $\{2 e\}$ denote two edges.

- The graph $K_{n}-\{e\}$ has a distance magic labeling if and only if $n=1,2$, or 3 .

- The graph $K_{n}-\{2 e\},(n \geq 3)$ has a distance magic labeling if and only if $n=4$ or 5 and $\{2 e\}$ is a 2-matching. 
Theorem 2.7. [40]

- The path $P_{n}$ on $n$ vertices is a distance magic graph if and only if $n=1$ or $n=3$.

- The cycle $C_{n}$ of length $n$ is a distance magic graph if and only if $n=4$.

- The complete graph $K_{n}$ on $n$ vertices is a distance magic graph if and only if $n=1$.

- The wheel $W_{n}(n \geq 4)$, formed by joining the vertices of $C_{n-1}$ to an extra center point, is a distance magic graph if and only if $n=5$.

- The tree $T$ is a distance magic graph if and only if $T=P_{1}$ or $T=P_{3}$.

Proof. Consider the possible graphs named above. Labeling $P_{1}=K_{1}$ is trivial. The other possible graphs are pictured in Figure 2.1. The impossibility of a distance magic

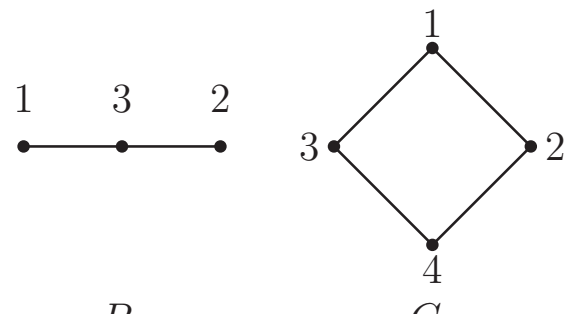

$P_{3}$
$C_{4}$

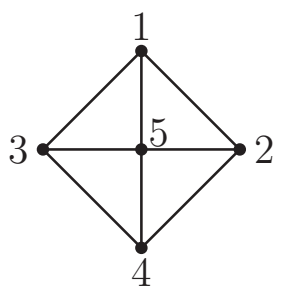

$W_{5}$

Figure 2.1: Basic Graphs

labeling of other paths, cycles, complete graphs, wheels, and trees follows directly from Theorem 2.5.

Theorem 2.8. [43], [54] Let $x, y, z \in V$. If $G$ is distance magic, $\operatorname{deg}(x)=\operatorname{deg}(y)=$ $\operatorname{deg}(z)=2$ and $y$ is adjacent to $x$ and $z$, then either $G$ is isomorphic to $C_{4}$ or $G$ contains a component isomorphic to $C_{4}$.

Corollary 2.9. [35], [55] If $C$ is a cycle component of a distance magic graph $G$, then $C$ is a 4-cycle.

Corollary 2.10. [35], [55] If a graph $G$ has a path $(v, w, x, y, z)$ with $\operatorname{deg}(w)=$ $\operatorname{deg}(y)=2$, then $G$ is not a distance magic graph.

Let $\delta(G)$ denote the least degree of any vertex in $G$ and let $\Delta(G)$ denote the maximum degree of any vertex in $G$. A perfect matching $M$ is a set of pairwise nonadjacent edges such that every vertex from the original graph is incident to exactly one edge in the matching. 
Theorem 2.11. [8] Let $G$ be any graph of order $n$ with $\Delta(G)=n-1$. Then $G$ is a distance magic graph if and only if $n$ is odd and $G \cong\left(K_{n-1}-M\right)+K_{1}$ where $M$ is a perfect matching in $K_{n-1}$.

Theorem 2.12. [54] If $G$ is a nontrivial distance magic graph and $\delta(G)=1$, then either $G$ is isomorphic to $P_{3}$ or $G$ contains exactly one component isomorphic to $P_{3}$ and all other components are isomorphic to $K_{2,2}=C_{4}$.

Theorem 2.13. [7], [35], [55] Let $G$ be a nontrivial distance magic graph of order $n$ with magic constant $k$. Then the following are equivalent.

- $k=n$

- $\delta(G)=1$

- Either $G$ is isomorphic to $P_{3}$ or $G$ contains exactly one component isomorphic to $P_{3}$ and all other components are isomorphic to $K_{2,2} \cong C_{4}$.

The symmetric difference of two sets is the set of elements that are in one but not both sets. Let $A \triangle B$ denote the symmetric difference of sets $A$ and $B$.

Theorem 2.14. [35], [55] Let $u$ and $v$ be vertices of a distance magic graph $G$. Then $\left|N_{G}(u) \triangle N_{G}(v)\right|=0$ or $\geq 3$.

Corollary 2.15. [35], [55] Let $G$ be a graph of order $n$ which has two vertices of degree $n-1$. Then $G$ is not a distance magic graph.

Lemma 2.16. [40] Let $G$ be a graph of order $n$. If $\Delta(G)(\Delta(G)+1)>\delta(G)(2 n-$ $\delta(G)+1)$ then $G$ does not have a distance magic labeling.

The following table, compiled from [8], characterizes all distance magic graphs of orders 4 through 7 .

\begin{tabular}{|c|c|}
\hline Order & Isomorphic to \\
\hline 4 & $C_{4}$ \\
\hline 5 & $W_{5}$ \\
\hline 6 & $K_{6}-M$ \\
\hline 7 & $P_{3} \cup C_{4}, K_{3,4}$, or $\left(K_{6}-M\right)+K_{1}$ \\
\hline
\end{tabular}

\subsection{Regular Graphs}

An $r$-regular graph is a graph in which every vertex is adjacent to $r$ other vertices. For example, the sample distance magic graph in the introduction was a 4-regular graph on 6 vertices.

Theorem 2.17. 
- [37] There exists a 4-regular distance magic graph of odd order $n$ if and only if $n \geq 17$.

- [39] There exists a 6-regular distance magic graph of odd order $n$ if and only if $n=9$ or $n \geq 13$.

- [39] There exists an 8-regular distance magic graph of odd order $n$ if and only if $n \geq 15$.

- [39] There exists a 10-regular distance magic graph of odd order $n$ if and only if $n \geq 15$.

- [39] There exists a 12-regular distance magic graph of odd order $n$ if and only if $n \geq 15$.

Theorem 2.18. [27] For $n$ even, an $r$-regular distance magic graph with $n$ vertices exists if and only if $2 \leq r \leq n-2, r \equiv 0(\bmod 2)$ and either $n \equiv 0(\bmod 4)$ or $n \equiv r+2 \equiv 2(\bmod 4)$.

Theorem 2.19. [21] Let $n, q$ be odd integers and $s$ an integer, $q \geq 3, s \geq 1$. Let $r=2^{s} q, q \mid n$, and $n \geq r+q$. Then, an $r$-regular distance magic graph of order $n$ exists.

Theorem 2.20. [38] Let $G$ be an r-regular distance magic graph of odd order $n$. There exists an $r$-regular distance magic graph with $m$ vertices for all odd $m=n+2 t$, if $2 t \geq r+2$ and if not both $\frac{r}{2}$ and $t$ are odd.

Theorem 2.21. [21] Let $n, q$ be odd integers and $s$ an integer, $q \geq 3, s \geq 1$. Let $r=2^{s} q, q \nmid n$, and $n \geq \frac{7 r+4}{2}$. Then, an $r$-regular distance magic graph of order $n$ exists.

Proposition 2.22. [21] Let $n, q>1$ be odd, and $s \geq 1$, and $r=2^{s} q$. Then, an $r$-regular distance magic graph of with $m$ vertices exists whenever $r<\frac{2}{7}(m-2)$.

Let $C_{n}\left(s_{1}, s_{2}, \ldots, s_{m}\right)$ denote the circulant graph on $n$ vertices with vertex set $V=\left\{x_{0}, x_{1}, \ldots, x_{n-1}\right\}$ and $\left\{x_{i}, x_{j}\right\}$ is an edge if and only if

$$
i-j \in\left\{ \pm s_{1}, \pm s_{2}, \ldots, \pm s_{m}\right\}
$$

For another interpretation of circulant graphs, see the Other Distances section.

Observation 2.23. [17] If $C_{n}(1, d)$ is a distance magic circulant graph, then $n \equiv 0$ $(\bmod 2)$. Moreover, when $d$ is odd, then $n \equiv 0(\bmod 8)$.

For example, consider $C_{8}(1,3)$ as shown in Figure 2.2. The magic constant $k=18$.

Observation 2.24. [17] A graph $C_{2 d+2}(1, d)$ is distance magic. 


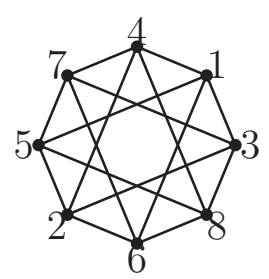

Figure 2.2: Labeling of $C_{8}(1,3)$

Theorem 2.25. [17] If $d$ is odd, then $C_{n}(1, d)$ is a distance magic graph if and only if $d^{2}-1 \equiv 0(\bmod n), \frac{n}{\operatorname{gcd}(n, d+1)} \equiv 0(\bmod 2)$, and $\frac{n}{\operatorname{gcd}(n, d-1)} \equiv 0(\bmod 2)$.

Observation 2.26. [17] If $d$ is even, then $C_{2\left(d^{2}-1\right)}(1, d)$ is distance magic.

A hypergraph $H$ is a pair $H=(V, E)$ where $V$ is a set of vertices and $E$ is a set of non-empty subsets of $V$ called hyperedges. If all edges have the same cardinality $t$ (each edge is incident to $t$ vertices), the hypergraph is $t$-uniform. Notice, in a hypergraph, an edge can connect any number of vertices and thus generalizes the concept of a "graph."

If hypergraph $H$ allows a distance magic labeling, we say $H$ is a distance magic hypergraph.

We define the $d$ th power of a graph $G$, denoted $G^{d}$, as the graph with the same set of vertices as $G$ and an edge between two vertices if and only if there is a path of length at most $d$ between them.

The $(r, t)$-hypercycle, $1 \leq r \leq t-1$ is defined as a $t$-uniform hypergraph whose vertices can be ordered cyclically in such a way that the edges are segments of that cyclic order and every two consecutive edges share exactly $r$ vertices. [36]

Theorem 2.27. [13] If $t>2$ and $r \leq \frac{t}{2}$, then an $(r, t)$-hypercycle is not distance magic.

Theorem 2.28. [13] If $t$ is even, then a $(t-2, t)$-hypercycle is not distance magic.

Theorem 2.29. [13] If $t \in\{3,4,6\}$, then an $(r, t)$-hypercycle of order $n$ is distance magic if and only if $r=t-1$ and one of the following conditions hold:

- $r=2$ and $n=6$,

- $r=3$ and $n=8$ or $n=24$,

- $r=5$ and $n \in\{12,20,60\}$.

Corollary 2.30. [13] Let $H$ be a $(t-1, t)$-hypercycle of order $n$.

- If $t$ is even, then $H$ is a distance magic hypergraph if and only if $2 t(t-1) \equiv 0$ $(\bmod n), n>2 t-1$, and $\frac{n}{\operatorname{gcd}(n, t)} \equiv 0(\bmod 2)$. 
- If $H$ is a distance magic hypergraph, then $n$ is even.

Notice the circulant graph $C_{n}(1,2, \ldots, d)$ is a $2 d$-regular graph that could also be written $C_{n}^{d}$ with our new notation. In addition, if $H$ is a $(t-1, t)$-hypercycle, then for graph $G_{H}$ with the same vertices and edges as hypergraph $H, G_{H} \cong C_{n}^{t-1}$. [13]

Theorem 2.31. [13] If $d$ is odd, then $C_{n}^{d}$ is a distance magic graph if and only if $2 d(d+1) \equiv 0(\bmod n), n \geq 2 d+2$, and $\frac{n}{\operatorname{gcd}(n, d+1)} \equiv 0(\bmod 2)$.

Lemma 2.32. [13] If $n=2 d+2$, then $C_{n}^{d}$ is a distance magic graph.

Theorem 2.33. [13], [51] The graph $C_{n}^{2}$ is not a distance magic graph unless $n=6$.

Theorem 2.34. [51]

$P_{n}^{k}$ has a distance magic labeling if and only if $n=1$ or 3 and $k=1$.

\subsection{Multipartite Graphs}

A multipartite graph is a graph whose vertex set can be partitioned into subsets, or parts, in such a way that no edge has both ends in the same part. [11]

Just as magic squares motivate distance magic graphs of a single part, magic rectangles motivate the study of distance magic multipartite graphs.

A magic rectangle $A=\left(a_{i j}\right)$ of size $m \times n$ is an $m \times n$ array whose entries are $\{1,2, \ldots, m n\}$, each appearing once, with all its row sums equal and with all its column sums equal. Because the sum of all entries is $\frac{m n(m n+1)}{2}$, we can calculate the sum of each column and the sum of each row easily. Namely,

$$
\sum_{i=1}^{m} a_{i j}=\frac{n(m n+1)}{2}
$$

for all $j$ yields the sum of each row and

$$
\sum_{j=1}^{n} a_{i j}=\frac{m(m n+1)}{2}
$$

for all $i$ yields the sum of each column.

Thus $m$ and $n$ must both be even or both be odd; that is they must have the same parity. [7] Such rectangles exist with few exceptions as shown by the next theorem.

Theorem 2.35. [33], [34] An $m \times n$ magic rectangle exists if and only if $m, n>$ $1, m n>4$, and $m \equiv n(\bmod 2)$. 
However, the nonexistence of a magic rectangle does not always mean the corresponding distance magic graph does not exist. For example, $K_{2,2}$ can be labeled $\{1,4\},\{2,3\}$ even though there is no $2 \times 2$ magic rectangle.

We now consider multipartite graphs of increasing size.

Theorem 2.36. [40] Let $1 \leq n_{1} \leq n_{2}$ where and let $s_{i}=\sum_{j=1}^{i} n_{j}$. There exists a distance magic labeling of the complete bipartite graph $K_{n_{1}, n_{2}}$ if and only if the following conditions hold:

- $n_{2} \geq 2$,

- $n(n+1) \equiv 0(\bmod 4)$, where $n=s_{2}=\left|V\left(K_{n_{1}, n_{2}}\right)\right|$, and

- $\sum_{j=1}^{s_{i}}(n+1-j) \geq \frac{i n(n+1)}{4}$ for $i=\{1,2\}$.

Let $\theta(n)$ be the largest value of $s$ such that $1+2+\cdots+s=\frac{s(s+1)}{2} \leq \frac{n(n+1)}{4}$.

Theorem 2.37. [2] If the complete bipartite graph $K_{n_{1}, n_{2}}$ is a distance magic graph, then $n \equiv 0$ or $3(\bmod 4)$ and $\left\lceil\frac{n}{\sqrt{2}}\right\rceil-2 \leq \theta(n)<\left\lceil\frac{n}{\sqrt{2}}\right\rceil$, where $n=n_{1}+n_{2}$.

Theorem 2.38. [2] The complete bipartite graph $K_{n_{1}, n_{2}}$ is a distance magic graph if and only if $n \equiv 0$ or $3(\bmod 4)$ where $n_{1}+n_{2}=n$ and $\frac{n}{2} \leq n_{1} \leq \theta(n)$.

Beena obtained the same results, which were formatted as follows.

Theorem 2.39. [9] Let $m$ and $n$ be two positive integers such that $m \leq n$. The complete bipartite graph $K_{m, n}$ is a distance magic graph if and only if

- $m+n \equiv 0$ or $3(\bmod 4)$ and

- either $n \leq\left\lfloor(1+\sqrt{2}) m-\frac{1}{2}\right\rfloor$ or $2(2 n+1)^{2}-(2 m+2 n+1)^{2}=1$.

We illustrate these theorems with the following example. Consider $K_{7,12}$. For partite sets $V_{1}$ and $V_{2}$, one labeling of $K_{7,12}$ is

$$
V_{1}=\{8,12,13,14,15,16,17\}, V_{2}=\{1,2,3,4,5,6,7,9,10,11,18,19\} .
$$

This produces a magic constant of $k=95$.

Let $t G$ denote the graph obtained by taking $t$ disconnected copies of the graph $G$.

Theorem 2.40. [47] Let $m$ and $n$ be two positive even integers such that $m \leq n$. The graph $t K_{m, n}$ is distance magic if and only if the conditions hold:

- $m+n \equiv 0(\bmod 4)$

- $1=2(2 t n+1)^{2}-(2 t m+2 t n+1)^{2}$ or $m \geq(\sqrt{2}-1) n+\frac{\sqrt{2}-1}{2 t}$.

Corollary 2.41. [35], [54], [55] Any complete multipartite graph with two partite sets of cardinality 1 is not a distance magic graph. 
Theorem 2.42. [40] Let $1 \leq n_{1} \leq n_{2} \leq n_{3}$ and let $s_{i}=\sum_{j=1}^{i} n_{j}$. There exists a labeling of the complete tripartite graph $K_{n_{1}, n_{2}, n_{3}}$ if and only if the following conditions hold:

- $n_{2} \geq 2$,

- $n(n+1) \equiv 0(\bmod 6)$, where $n=s_{3}=\left|V\left(K_{n_{1}, n_{2}, n_{3}}\right)\right|$, and

- $\sum_{j=1}^{s_{i}}(n+1-j) \geq \frac{i n(n+1)}{6}$ for $i=\{1,2,3\}$.

Theorem 2.43. [51]

- If $n \geq 6$, the graph $K_{1, s, t}, 1+s+t=n$ is not distance magic.

- The complete four partite graph $K_{1, q, s, t}$ has a distance magic labeling if and only if $q=s=t=2$.

- The complete four partite graph $K_{2, q, s, t}$ has a distance magic labeling if and only if $q=s=t=2$.

Theorem 2.44. [40] Let $m, n>1$. The complete $m$ partite graph with each part of size $n$ is distance magic if and only if either $n$ is even or both $n$ and $m$ are odd.

Theorem 2.45. [20] Let $m$ and $n$ be two positive even integers such that $m \leq n$. The graph $K_{\underbrace{m, \ldots, m}_{t}} \underbrace{n, \ldots, n}_{t}$ is distance magic if and only if the conditions hold:

- $m+n \equiv 0(\bmod 4)$

- $1=2(2 t n+1)^{2}-(2 t m+2 t n+1)^{2}$ or $m \geq(\sqrt{2}-1) n+\frac{\sqrt{2}-1}{2 t}$.

The graph $K_{4,4,8,8}$ meets the conditions of the previous theorem. For partite sets $V_{1}, V_{2}, V_{3}$ and $V_{4}$, one labeling is $V_{1}=\{15,17,19,24\}, V_{2}=\{14,18,20,23\}$, $V_{3}=\{2,4,5,8,9,12,13,22\}$, and $V_{4}=\{1,3,6,7,10,11,16,21\}$. This produces a magic constant of $k=225$.

Theorem 2.46. [52]

- If $n$ is even or $m n p$ is odd, $m \geq 1, n>1$, and $p>1$, then $m K_{\underbrace{p, p, \ldots, p}_{n}}^{p a s}$ a distance magic labeling.

- If $n p$ is odd, $p \equiv 3(\bmod 4)$ and $m$ is even, then $m \underbrace{p, p, \ldots, p}_{n}$ does not have a distance magic labeling. 


\subsection{Graph Products}

We list results on the four standard graph products: the lexicographic product, direct product, Cartesian product, and strong product.

\subsubsection{Lexicographic Product}

We first discuss the lexicographic product of $G$ and $H$, also called the composition of graphs $G$ and $H$, denoted $G \circ H$ with vertex set $V(G) \times V(H)$. (Some authors use $G[H]$ to denote this product.) $G \circ H$ is created by replacing every vertex of $G$ with a copy of $H$. Thus, vertices $(g, h)$ and $\left(g^{\prime}, h^{\prime}\right)$ in $G \circ H$ are adjacent if and only if either $g$ is adjacent to $g^{\prime}$ in $G$ or $g=g^{\prime}$ and $h$ is adjacent to $h^{\prime}$ in $H$. [32]

Theorem 2.47. [52] Let $r \geq 1, n \geq 3, G$ be an $r$-regular graph and $C_{n}$ be the cycle of length $n$. The graph $G \circ C_{n}$ admits a distance magic labeling if and only if $n=4$.

For example, $C_{3} \circ C_{4}$, is a distance magic graph with magic constant $k=65$ as shown in Figure 2.3

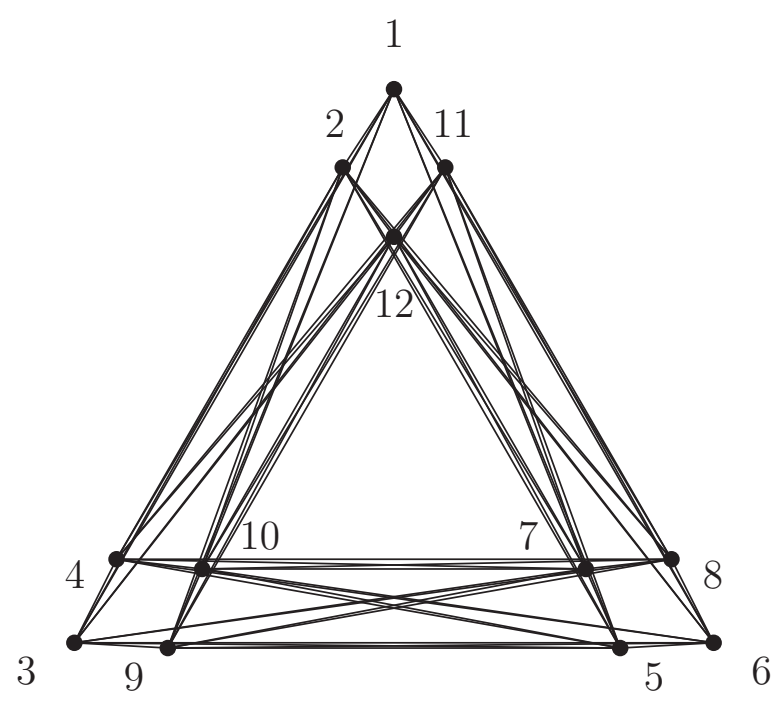

Figure 2.3: Labeling of $C_{3} \circ C_{4}$

Theorem 2.48. [4] Let $m$ and $n$ be integers such that $1 \leq m<n$. Then $K_{m, n} \circ C_{4}$ is distance magic if and only if the following conditions hold:

- The numbers $a=\frac{(m+n)(4 m+4 n+1)(2 m-1)}{4 m n-m-n}$ and $b=\frac{(m+n)(4 m+4 n+1)(2 n-1)}{4 m n-m-n}$ are integers.

- There exist integers $p, q, t \geq 1$, such that $p+q=(b-a), 4 n=p t$, and $4 m=q t$. 
There are very few pairs $(m, n)$ that satisfy this theorem. For $1 \leq m<n \leq 80000$, only the following pairs $(m, n)$ yield a distance magic $K_{m, n} \circ C_{4}$ graph: $(9,21),(20,32)$, $(428,548),(2328,2748),(6408,10368),(7592,8600),(10098,24378),(18860,20840)$, $(39540,42972)$

(73808, 79268). [4]

Let $\bar{G}$ denote the complement of graph $G$.

Theorem 2.49. [52] Let $m \geq 1, n>1$, and $p \geq 3$. Then $m C_{p} \circ \bar{K}_{n}$ has a distance magic labeling if and only if either $n$ is even or mnp is odd or $n$ is odd and $p \equiv 0$ $(\bmod 4)$.

Theorem 2.50. [26] The graph $m K_{p} \circ \bar{K}_{n}$, where $n p$ is odd and $m$ is even, $p>1$, $m \geq 2$, is distance magic if and only if $p \equiv 3(\bmod 4)$.

Theorem 2.51. [26] Let $n$ be odd, $m \equiv r \equiv 2(\bmod 4)$, and $G$ be an $r$-regular graph with $m$ vertices. Then $G \circ \bar{K}_{n}$ is not distance magic.

Theorem 2.52. [40] If $G=H \circ \bar{K}_{2 m}$, where $H$ is an $r$-regular graph, then $G$ has a distance magic labeling.

Theorem 2.53. [38] An (n-3)-regular distance magic graph $G$ with $n$ vertices exists if and only if $n \equiv 3(\bmod 6)$. Further $G$ is isomorphic to $K_{n / 3} \circ \bar{K}_{3}$.

Theorem 2.54. [26] Let $G$ be an arbitrary $r$-regular graph with an odd number of vertices and let $n$ be an odd positive integer. Then $r$ is even and the graph $G \circ \bar{K}_{n}$ is distance magic.

Consider the Dutch windmill graph or friendship graph $C_{m}^{(t)}$. This graph is obtained by taking $t>1$ copies of the cycle $C_{m}$ with a single vertex in common. [28] Figure 2.4 displays $C_{3}^{(5)}$.

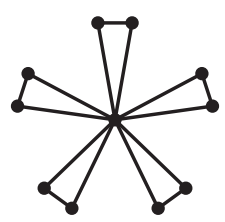

Figure 2.4: Dutch Windmill Graph $C_{3}^{(5)}$

Observation 2.55. [16] There does not exist a distance magic graph $C_{4}^{(t)} \circ C_{4}$.

Theorem 2.56. [4] The graph $C_{3}^{(t)} \circ C_{4}$ is not distance magic for any $t>1$. 


\subsubsection{Direct Product}

The direct product, denoted $G \times H$, is a graph with vertex set $V(G) \times V(H)$. Vertices $(g, h)$ and $\left(g^{\prime}, h^{\prime}\right)$ in $G \times H$ are adjacent if and only if $g$ is adjacent to $g^{\prime}$ in $G$ and $h$ is adjacent to $h^{\prime}$ in $H$. [32]

Corollary 2.57. [6] Let $G$ be an arbitrary regular graph. Then $G \times C_{4}$ is distance magic.

Theorem 2.58. [6] A graph $C_{m} \times C_{n}$ is distance magic if and only if $n=4$ or $m=4$ or $m, n \equiv 0(\bmod 4)$.

For example, by the above theorems, we know $C_{3} \times C_{4}$ is a distance magic graph. We illustrate this with Figure 2.5. The magic constant is 26.

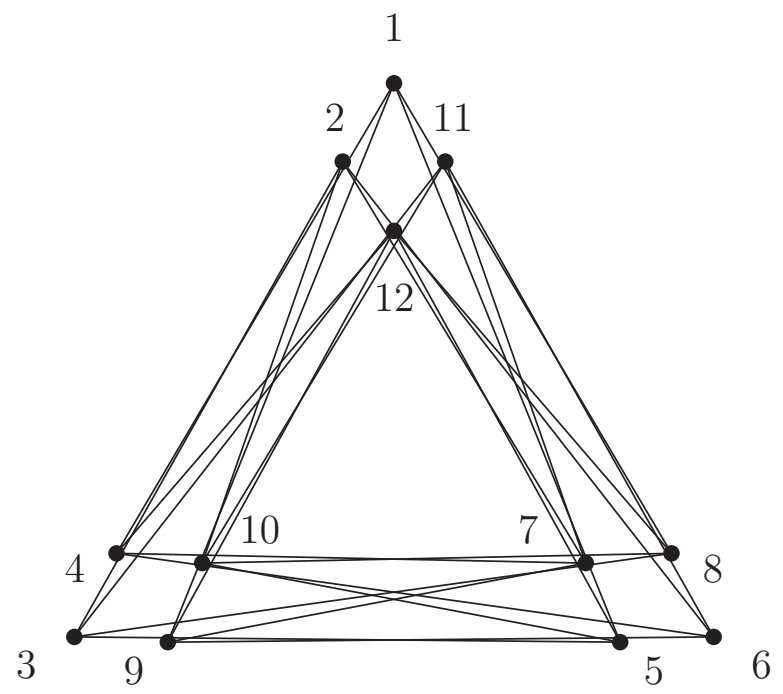

Figure 2.5: Labeling of $C_{3} \times C_{4}$

Theorem 2.59. [5] Let $m$ and $n$ be two positive integers such that $m \leq n$. The graph $K_{m, n} \times C_{4}$ is a distance magic graph if and only if the following conditions hold:

- $m+n \equiv 0(\bmod 2)$ and

- $m \geq \frac{\sqrt{2(8 n+1)^{2}-1}-1}{8}-n$.

Theorem 2.60. [20] Let $m$ and $n$ be two positive even integers such that $m \leq n$. The graph $K_{m, n} \times C_{4}$ is distance magic if and only if the conditions hold:

- $m+n \equiv 0(\bmod 2)$

- $1=2(8 n+1)^{2}-(8 m+8 n+1)^{2}$ or $m \geq(\sqrt{2}-1) n+\frac{\sqrt{2}-1}{8}$. 
To find $m$ and $n$ that satisfy the above, we must solve the Diophantine equation $\alpha=2(4 n+1)^{2}-(4 m+4 n+1)^{2}$ for $\alpha \in \mathbb{N}$. One such solution is $K_{102,246} \times C_{4}$. [20]

Theorem 2.61. [20] Consider friendship graph $C_{3}^{(t)}$. There does not exist a $C_{3}^{(t)} \times C_{4}$ distance magic graph.

\subsubsection{Cartesian Product}

The Cartesian product, denoted $G \square H$ is a graph with vertex set $V(G) \times V(H)$. Vertices $(g, h)$ and $\left(g^{\prime}, h^{\prime}\right)$ in $G \square H$ are adjacent if and only if $g=g^{\prime}$ and $h$ is adjacent to $h^{\prime}$ in $H$, or $h=h^{\prime}$ and $g$ is adjacent to $g^{\prime}$ in $G$. [32] For an illustration of the Cartesian product, see the Group distance magic section in Chapter 4.

Theorem 2.62. [9] Let $G_{1}$ and $G_{2}$ be connected graphs with $\delta\left(G_{i}\right)=1,\left|V\left(G_{i}\right)\right| \geq 3$ for $i=1,2$. Then $G_{1} \square G_{2}$ is not a distance magic graph.

\section{Theorem 2.63.}

- $[48] C_{n} \square C_{m}, n, m \geq 3$ is a distance magic graph if and only if $n=m \equiv 2$ $(\bmod 4)$.

- [9], [51] The Cartesian product $P_{m} \square P_{n}$ for $m \geq 2$ and $n \geq 2$ has a distance magic labeling if and only if $m=n=2$.

- [48], [51] The Cartesian product $K_{m} \square K_{n}, m, n \geq 2$ has a distance magic labeling if and only if $m=n=2$.

- [51] The Cartesian product $K_{m} \square K_{n, q}, n \leq q$, has a distance magic labeling if and only if $(m, n, q)=(2,1,1)$ or $(1,1,2)$.

- [51] The Cartesian product $K_{n} \square C_{m}$ has no distance magic labeling when $n$ is even.

- [51] The Cartesian product $K_{n} \square C_{3}$ has no distance magic labeling when $n$ is odd.

- [51] The Cartesian product $K_{m, n} \square P_{q}, m, n \geq 1$ and $q \geq 2$ has a distance magic labeling if and only if $m=n=1$, and $q=2$.

- [51] The Cartesian product $K_{n} \square P_{m}, n, m \geq 2$ has a distance magic labeling if and only if $n=m=2$.

- [51] The Cartesian product $C_{m} \square P_{n}, n$ an odd integer greater than 1 or $n \equiv 2$ (mod 4) has no distance magic labeling.

- [51] The Cartesian product $C_{m} \square K_{1, n}, n \geq 1$ has no distance magic labeling. 
- [51] The Cartesian product $C_{m} \square K_{n, n}$ where $n \neq 2$ and $m$ odd, has no distance magic labeling.

- [51] If $n$ is even and $m \equiv 1(\bmod 4)$, the Cartesian product graph $C_{m} \square K_{n, n+1}$ has no distance magic labeling.

\subsubsection{Strong Product}

The strong product, denoted $G \otimes H$ is a graph with vertex set $V(G) \times V(H)$. Vertices $(g, h)$ and $\left(g^{\prime}, h^{\prime}\right)$ in $G \otimes H$ are adjacent if and only if $g=g^{\prime}$ and $h$ is adjacent to $h^{\prime}$ in $H$, or $h=h^{\prime}$ and $g$ is adjacent to $g^{\prime}$ in $G$, or $g$ is adjacent to $g^{\prime}$ in $G$ and $h$ is adjacent to $h^{\prime}$ in $H$. [32]

Theorem 2.64. If $n>1$ and $n \not \equiv 0(\bmod 4), C_{n} \otimes C_{4}$ is not a distance magic graph.

Proof. If $n=1, C_{1} \otimes C_{4} \cong C_{4}$ which is distance magic by Theorem 2.7.

For $n=2, C_{2} \otimes C_{4}$ is a 5-regular graph, which is not distance magic by Theorem 2.3. (For a diagram, see Figure 2.6.)

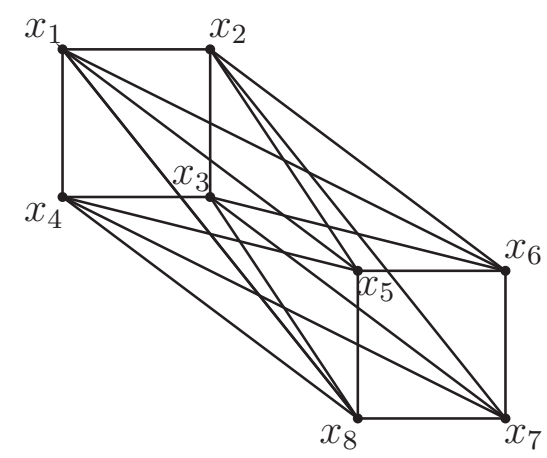

Figure 2.6: $C_{2} \otimes C_{4}$

For $n \geq 3$, consider labeling the vertices as described in Figure 2.7.

If $n=3$,

$$
N\left(x_{1}\right)=\left\{x_{2}, x_{4}, x_{5}, x_{6}, x_{8}, x_{9}, x_{10}, x_{12}\right\}
$$

and

$$
N\left(x_{5}\right)=\left\{x_{1}, x_{2}, x_{4}, x_{6}, x_{8}, x_{9}, x_{10}, x_{12}\right\} .
$$

(See Figure 2.8.) Because the weights must be equal in a distance magic graph, this implies $\ell\left(x_{5}\right)=\ell\left(x_{1}\right)$ which is impossible.

For $n \geq 5$, we consider the neighborhoods of the odd vertices. We have the following:

$$
\begin{aligned}
& N\left(x_{1}\right)=\left\{x_{2}, x_{4}, x_{5}, x_{6}, x_{8}, x_{4 n-3}, x_{4 n-2}, x_{4 n}\right\} \\
& N\left(x_{3}\right)=\left\{x_{2}, x_{4}, x_{6}, x_{7}, x_{8}, x_{4 n-2}, x_{4 n-1}, x_{4 n}\right\},
\end{aligned}
$$




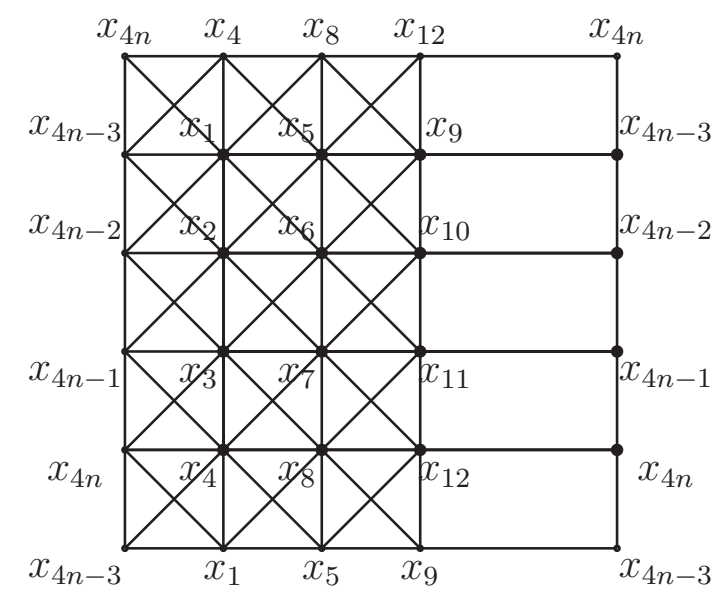

Figure 2.7: Vertex Layout for $C_{n} \otimes C_{4}$

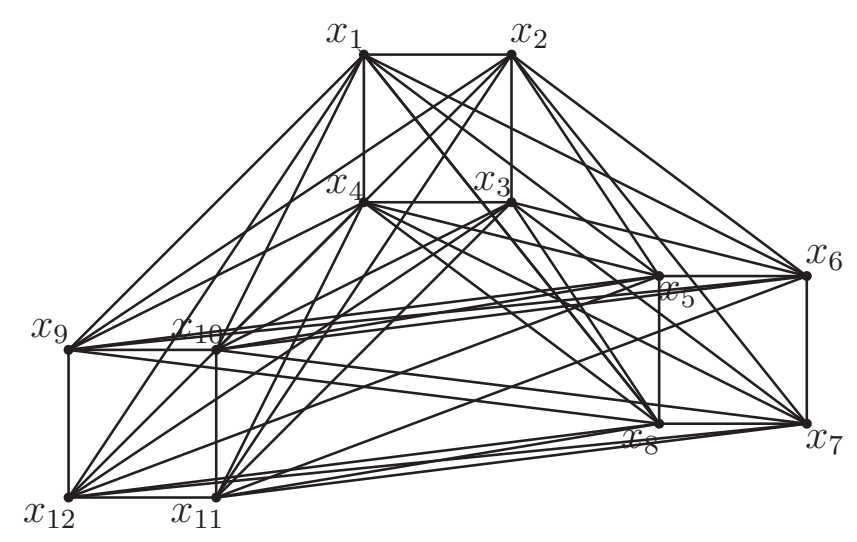

Figure 2.8: $C_{3} \otimes C_{4}$

which implies $\ell\left(x_{5}\right)+\ell\left(x_{4 n-3}\right)=\ell\left(x_{7}\right)+\ell\left(x_{4 n-1}\right)$. Similarly, we have

$$
\begin{gathered}
N\left(x_{5}\right)=\left\{x_{1}, x_{2}, x_{4}, x_{6}, x_{8}, x_{9}, x_{10}, x_{12}\right\} \\
N\left(x_{7}\right)=\left\{x_{2}, x_{3}, x_{4}, x_{6}, x_{8}, x_{10}, x_{11}, x_{12}\right\},
\end{gathered}
$$

which implies $\ell\left(x_{1}\right)+\ell\left(x_{9}\right)=\ell\left(x_{3}\right)+\ell\left(x_{11}\right)$. Continuing in the same manner for all odd vertices gives the final equation $\ell\left(x_{1}\right)+\ell\left(x_{4 n-7}\right)=\ell\left(x_{3}\right)+\ell\left(x_{4 n-5}\right)$.

Now, vertices from the same 4-cycle each appear in two such equations. If $n \equiv 3$ (mod 4$)$, by rearranging, we have a string of equations from every other 4-cycle of the form $\ell\left(x_{1}\right)-\ell\left(x_{3}\right)=\ell\left(x_{11}\right)-\ell\left(x_{9}\right)=\ell\left(x_{17}\right)-\ell\left(x_{19}\right)=\cdots=\ell\left(x_{4 n-1}\right)-\ell\left(x_{4 n-3}\right)=$ $\ell\left(x_{5}\right)-\ell\left(x_{7}\right)=\ell\left(x_{15}\right)-\ell\left(x_{13}\right)=\cdots=\ell\left(x_{4 n-7}\right)-\ell\left(x_{4 n-5}\right)=\ell\left(x_{3}\right)-\ell\left(x_{1}\right)$. Now, $\ell\left(x_{1}\right)-\ell\left(x_{3}\right)=\ell\left(x_{3}\right)-\ell\left(x_{1}\right)$ implies $\ell\left(x_{1}\right)=\ell\left(x_{3}\right)$, which is impossible in a distance magic graph.

If $n \equiv 1(\bmod 4)$, by rearranging, we have the string of equations $\ell\left(x_{1}\right)-\ell\left(x_{3}\right)=$ $\ell\left(x_{11}\right)-\ell\left(x_{9}\right)=\ell\left(x_{17}\right)-\ell\left(x_{19}\right)=\cdots=\ell\left(x_{4 n-3}\right)-\ell\left(x_{4 n-1}\right)=\ell\left(x_{7}\right)-\ell\left(x_{5}\right)=$ 


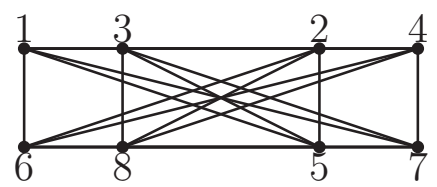

Figure 2.9: Join Graph $C_{4}+C_{4}$

$\ell\left(x_{13}\right)-\ell\left(x_{15}\right)=\cdots=\ell\left(x_{4 n-7}\right)-\ell\left(x_{4 n-5}\right)=\ell\left(x_{3}\right)-\ell\left(x_{1}\right)$, which provides the same problem as the previous case.

If $n \equiv 2(\bmod 4)$, by rearranging, we have the string of equations $\ell\left(x_{1}\right)-\ell\left(x_{3}\right)=$ $\ell\left(x_{11}\right)-\ell\left(x_{9}\right)=\ell\left(x_{17}\right)-\ell\left(x_{19}\right)=\cdots=\ell\left(x_{4 n-7}\right)-\ell\left(x_{4 n-5}\right)=\ell\left(x_{3}\right)-\ell\left(x_{1}\right)$, which provides the same problem as the previous cases.

Thus for $n>1$ and $n \not \equiv 0(\bmod 4), C_{n} \otimes C_{4}$ is not a distance magic graph.

\subsection{Join Graphs}

A join graph is formed by the union of two graphs plus all edges connecting the vertices of the first graph to the second graph. For example, see Figure 2.9 for a distance magic labeling of $C_{4}+C_{4}$ with magic constant $k=27$.

Theorem 2.65. [51]

- The join graph $C_{n}+P_{m}$ has a distance magic labeling if and only if $n=4$ and $m=1$ or 3 .

- The join graph $C_{m}+C_{n}, m, n \geq 3$ has a distance magic labeling if and only if $n=m=4$.

- The join graph $P_{m}+P_{n}$ has no distance magic labeling.

Theorem 2.66. [51]

- The join graph $K_{m, n}+P_{q}$ has a distance magic labeling if and only if $m=n=2$ and $q=1$ or 3 .

- The join graph $K_{m, n}+C_{q}$ has a distance magic labeling if and only if $m=1, n=$ 2 , and $q=4$ or $m=n=2$ and $q=4$.

Theorem 2.67. [51]

- The join graph $C_{m}+\bar{K}_{n}$ has a distance magic labeling if and only if $m=4$ and $n=1,2,4$, or 5 .

- The join graph $P_{n}+\bar{K}_{m}$ has a distance magic labeling if and only if $n=1$ or 3 , and $m=2$. 


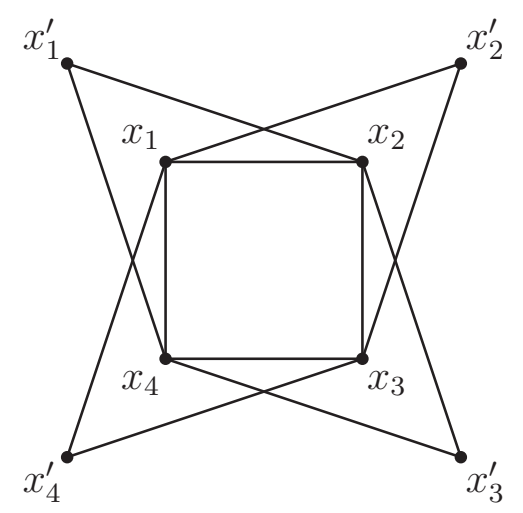

Figure 2.10: Splitting Graph $S^{\prime}\left(C_{4}\right)$

Theorem 2.68. [51]

- The join graph $K_{n}+G, n \geq 2$ has no distance magic labeling.

- The join graph $K_{n}+\bar{K}_{m}$ has a distance magic labeling if and only if $n=1$ and $m=2$.

- The join graph $K_{n}+C_{m}$ has a distance magic labeling if and only if $n=1$ and $m=4$.

- The join graph $K_{n}+P_{m}$ has no distance magic labeling.

- The join graph $K_{m, n}+K_{q}$ has a distance magic labeling if and only if $m=n=2$ and $q=1$.

\subsection{Splitting Graphs}

Let $S^{\prime}(G)$ represent the splitting graph of $G$. A splitting graph is obtained by adding a new vertex $x^{\prime}$ for every vertex $x$ in $G$ such that $x^{\prime}$ is adjacent to all vertices adjacent to $x$ in the original graph $G$. For example, the splitting graph $S^{\prime}\left(C_{4}\right)$ is pictured in Figure 2.10.

Theorem 2.69. [51] The splitting graph $S^{\prime}\left(K_{m, n}\right)$ has no distance magic labeling.

Theorem 2.70. The splitting graph $S^{\prime}(G)$ is distance magic if and only if $G \cong \bar{K}_{n}$.

Proof. Trivially, $S^{\prime}\left(\bar{K}_{n}\right)$ is distance magic with magic constant $k=0$.

Certainly for any vertex $g$ and its new vertex $g^{\prime}$ in the splitting graph, $N_{S^{\prime}(G)}\left(g^{\prime}\right) \subseteq$ $N_{S^{\prime}(G)}(g)$. Moreover, if $N_{S^{\prime}(G)}\left(g^{\prime}\right) \subsetneq N_{S^{\prime}(G)}(g)$, the extra vertices in the neighborhood of $g$ would have to have labels summing to 0 , which is impossible in a distance magic graph. For $N_{S^{\prime}(G)}\left(g^{\prime}\right)=N_{S^{\prime}(G)}(g), g$ cannot be adjacent to any vertex. Because $g$ 
is arbitrary, the only graphs satisfying this neighborhood restriction are of the form $S^{\prime}\left(\bar{K}_{n}\right)$.

\subsection{Embedding Theorems}

An embedding of a graph $H$ in graph $G$ is an isomorphism between $H$ and a subgraph of $G[11]$.

Theorem 2.71. [35], [55] Every graph is a subgraph of a distance magic graph.

An induced subgraph $H$ is a subgraph of original graph $G$ whose vertex set is a subset, $Y$, of the vertex set of $G$ and whose edge set consists of all edges of $G$ which have both ends in $Y$. [11]

Theorem 2.72. [2] Every graph $H$ is an induced subgraph of a regular distance magic graph.

Corollary 2.73. [2] There is no forbidden subgraph characterization for distance magic graphs.

The chromatic number of graph $H$ is the minimum number of colors in a proper coloring of graph $H$.

An Eulerian graph contains an Eulerian cycle. An Eulerian cycle begins and ends at the same vertex and uses each edge once.

Theorem 2.74. [46] Given any graph $H$, there is an Eulerian distance magic graph $G$ with chromatic number equal to $H$ such that $H$ is an induced subgraph of $G$.

Corollary 2.75. [7] The problem of deciding whether the chromatic number of an Eulerian distance magic graph is at least 3 is NP-complete.

\subsection{Matrix Representation}

Let $G=(V, E)$ be a graph of order $n$ with $V=\left\{v_{1}, v_{2}, \ldots, v_{n}\right\}$. Let $A=\left(a_{i, j}\right)$ be the adjacency matrix of $G$. Let $f: V \rightarrow\{1,2, \ldots, n\}$ be a bijection, which gives a labeling of the vertices of $G$. The matrix $A_{f}=\left(b_{i, j}\right)$ of the labeling $f$ is defined as follows:

$$
b_{i, j}= \begin{cases}a_{i, j} & \text { if } a_{i, j}=0 \\ f\left(v_{j}\right) & \text { if } a_{i, j}=1\end{cases}
$$

Notice, matrix $A_{f}$ is not symmetric, but $A_{f}$ is obtained from $A$ by multiplying the $i$ th column of $A$ by $f\left(v_{i}\right)$ for $i=\{1,2, \ldots, n\}$. In addition, for distance magic graph $G$ with magic constant $k, k$ is an eigenvalue of $A_{f}$. [7]

Corollary 2.76. [41] Let $G$ be any regular graph. If $A^{-1}$ exists, $G$ is not distance magic. 


\subsection{Lifted Magic Rectangles and $p$-regular graphs}

A lifted magic rectangle $\operatorname{LMR}(a, b ; \ell)$ is an $a \times b$ matrix whose entries are elements of $\{\ell+1, \ell+2, \cdots, \ell+a b\}$ each appearing once, such that the sum of each column is $\sigma(a, b ; \ell)=\frac{1}{2} a(a b+2 \ell+1)$ and the sum of each row is $\tau(a, b ; \ell)=\frac{1}{2}(a b+2 \ell+1)$. Consider $\operatorname{LMR}(a, b ; 0)$ and $p$-regular graph $H$ with $n^{\prime}$ vertices such that $n^{\prime}=b+d$, where $b=\left|V_{1}(H)\right|$ and $d=\left|V_{2}(H)\right|$ for $V_{1}, V_{2} \subset V(H)$ such that $V_{1}=\left\{x_{1}, x_{2}, \cdots, x_{b}\right\}$ and $V_{2}=\left\{y_{1}, y_{2}, \cdots, y_{d}\right\}$ form a partition of $V(H)$.

Consider graph $G$ arising from $H$ by expanding each vertex $x_{i} \in V_{1}(H)$ into a set $X_{i}$ of $a$ independent vertices $\left\{x_{i 1}, x_{i 2}, \cdots, x_{i a}\right\}$ and similarly expanding each $y_{j} \in V_{2}(H)$ into a set of $c$ independent vertices $\left\{y_{j 1}, y_{j 2}, \cdots, y_{j c}\right\}$. We denote this graph by $G=H[b \times a, d \times c]$. Further, each edge $x_{i} x_{j}$ between two vertices of $V_{1}(H)$ are replaced by $a^{2}$ edges of $K_{a, a}$ and each edge $y_{i} y_{j}$ between two vertices of $V_{2}(H)$ are replaced by $c^{2}$ edges of $K_{c, c}$. Also any edge $x_{i} y_{j}$ between a vertex in $V_{1}(H)$ and a vertex in $V_{2}(H)$ is replaced by ac edges of $K_{a, c}$. Denote $V_{1}(G)=X_{1} \bigcup X_{2} \bigcup \cdots \cup X_{b}$ and $V_{2}(G)=Y_{1} \cup Y_{2} \cup \cdots \cup Y_{d \cdot}[7]$

Lemma 2.77. [54] Let $a, b, c, d$ be positive integers such that $a>c$ and both $\operatorname{LMR}(a, b ; 0)$ and $\operatorname{LMR}(c, d ; a b)$ exist. Then $\sigma(a, b ; 0)=\sigma(c, d ; a b)$ if and only if $d=\frac{a^{2} b-2 a b c+a-c}{c^{2}}$.

Lemma 2.78. [54] Let $a, b, c, d$ be positive integers such that $\sigma(a, b ; 0)=\sigma(2, d ; a b)$ and both $\operatorname{LMR}(a, b ; 0)$ and $\operatorname{LMR}(2, d ; a b)$ exist. Then either $a \equiv 2(\bmod 4)$ or $a$ is odd and $a \equiv b(\bmod 4)$ and $a \geq 5$.

Theorem 2.79. [54] Let $H$ be a p-regular graph on $b+d$ vertices and $G=H[b \times$ $a, d \times c]$ be a graph with $a, b, c, d$ satisfying conditions

- $a>c$

- both $\operatorname{LMR}(a, b ; 0)$ and $\operatorname{LMR}(c, d ; a b)$ exist, and

- $d=\frac{a^{2} b-2 a b c+a-c}{c^{2}}$.

Then $G$ is a distance magic graph.

\subsection{Distance Magic Constants}

To this point, we have looked at graph structures that permit distance magic labelings. Some results are also known about the magic constants related to these graphs.

\section{Theorem 2.80.}

- [7], [44] For any distance magic graph $G$, the distance magic constant is unique. 
- [8] For any odd integer $k \geq 3$, there exists a distance magic graph with magic constant $k$.

- [8] There is no distance magic graph with magic constant $k=4,6,8$, or 12 .

- [8] Any distance magic graph with magic constant $k=10$ is isomorphic to the wheel $W_{5}$.

- [8] There is no distance magic graph of order $n$ with magic constant $k=n+2$.

- [35] A graph $G$ of order $n$ is a distance magic graph with magic constant $k=n+1$ if and only if $G=t C_{4}$.

Theorem 2.81. [8] Let $n$ be any positive integer with $n \equiv 3(\bmod 4)$. Then for each positive integer $j$ with $1 \leq j \leq \frac{n-1}{2}$, there exists a distance magic graph $G_{j}$ of order $n$ with magic constant $j n$.

Theorem 2.82. [8] Let $n$ be any positive integer with $n \equiv 1(\bmod 4)$. Then for each even positive integer $j$ with $2 \leq j \leq \frac{n-1}{2}$, there exists a distance magic graph $G_{j}$ of order $n$ with magic constant $j n$.

Observation 2.83. [8] There exist distance magic graphs of order $n$ whose distance magic constant is not a multiple of $n$.

For example, consider $K_{3,3,3,3,3}$ where each partite set is labeled as follows: $\{1,8,15\},\{3,7,10\},\{5,6,13\},\{2,10,12\},\{4,9,11\}$. This graph of order 15 has magic constant 96 .

Corollary 2.84. [37] There exists a 4-regular distance magic graph with magic constant $k=2^{t}$ for every $t \geq 6$. 


\section{Chapter 3}

\section{Related Graph Labelings}

The graph labelings discussed in this chapter are closely related to distance magic labelings.

\subsection{Balanced Distance Magic Graphs}

A balanced distance magic graph is a distance magic graph $G$ of order $n$ such that there exists a bijection $\ell: V(G) \rightarrow\{1,2, \ldots, n\}$, where for every vertex $x \in V(G)$, if $u \in N_{G}(x)$ with $\ell(u)=i$, there exists $v \in N_{G}(x), v \neq u$, with $\ell(v)=|V(G)|+1-i$. We call $u$ and $v$ twin vertices or twins. [6]

Proposition 3.1. [6] Let $V(G)=n$. Graph $G$ is balanced distance magic if and only if $G$ is regular, and $V(G)$ can be partitioned in pairs $\left(u_{i}, v_{i}\right), i \in\left\{1, \ldots, \frac{n}{2}\right\}$ such that $N\left(u_{i}\right)=N\left(v_{i}\right)$ for all $i$.

Theorem 3.2. [6] Let $G$ be a regular graph and $H$ a graph not isomorphic to $\overline{K_{n}}$ where $n$ is odd. Then $G \circ H$ is a balanced distance magic graph if and only if $H$ is a balanced distance magic graph.

Theorem 3.3. [6] The direct product $G \times H$ is balanced distance magic if and only if one of the graphs $G$ and $H$ is balanced distance magic and the other one is regular.

Theorem 3.4. [6] $C_{m} \times C_{n}$ is balanced distance magic if and only if $n=4$ or $m=4$.

\subsection{Other Distances}

In this section, we explore theorems that justify the name "distance" magic because they apply to distances other than 1. As noted in Section 2.2, powers of graphs and circulant graphs can also be interpreted as other distances from a vertex. 


\subsubsection{Closed Distance Magic Graphs}

So far, we have considered distance magic or sigma graphs, which calculate the weight of each vertex using open neighborhoods. However, we can also consider closed neighborhoods in our definition. These modified graphs are called closed distance magic or $\Sigma^{\prime}$ graphs and are defined as follows.

Let $G=(V, E)$ be a graph on $n$ vertices. Let $N_{G}[x]=N_{G}(x) \bigcup\{x\}$ be the closed neighborhood of $x$ in $G$. The graph $G$ is said to be a closed distance magic graph if there is a bijection $\ell: V(G) \rightarrow\{1,2, \ldots, n\}$ with the property that there is a positive integer $k^{\prime}$, the magic constant, such that for every $x \in V(G)$, the weight $w^{\prime}(x)=$ $\ell(x)+\sum_{y \in N_{G}(x)} \ell(y)=k^{\prime}$. (Isolated vertices are permitted in these graphs.) [9]

This altered definition leads to some results related to distance magic graphs.

Observation 3.5. [1] If $G$ is an r-regular closed distance magic graph on $n$ vertices, then $k^{\prime}=\frac{(r+1)(n+1)}{2}$.

Theorem 3.6. [35], [41] Let $G=(V, E)$ be a graph on $n$ vertices and $\ell: V(G) \rightarrow$ $\{1,2, \ldots, n\}$ be a labeling. Then $\ell$ is a distance magic labeling for $G$ with magic constant $k$ if and only if $\ell$ is a closed distance magic labeling for the complement $\bar{G}$ with magic constant $k^{\prime}=\frac{n(n+1)}{2}-k$.

Lemma 3.7. [41] There does not exist a graph of even order that admits both distance magic and closed distance magic labelings.

Theorem 3.8. [9] $K_{m} \cup K_{n}$ is a closed distance magic graph if and only if $m+n \equiv 0$ or $3(\bmod 4)$ and either $n \leq\left\lfloor(1+\sqrt{2}) m-\frac{1}{2}\right\rfloor$ or $2(2 n+1)^{2}-(2 m+2 n+1)^{2}=1$.

S. Beena established a number of structural constraints for closed distance magic graphs.

Theorem 3.9. [9]

- Let $G$ be a closed distance magic graph on $n$ vertices. $\delta(G)=0$ if and only if $G \cong K_{1} \bigcup r K_{2}$ where $r=\frac{n-1}{2}$.

- Let $G$ be a closed distance magic graph on $n$ vertices. $\Delta(G)=n-1$ if and only if $G \cong K_{n}$.

- Let $x$ and $y$ be vertices of a closed distance magic graph $G$. Then $\left|N_{G}[x] \triangle N_{G}[y]\right|=0$ or $\geq 3$.

- If two adjacent vertices of degree $n$ of a graph $G$ have exactly $n-1$ common neighbors, then $G$ cannot be a closed distance magic graph.

- Let $G$ be a closed distance magic graph and let $H$ be a complete subgraph of $G$ of order $n$. Let $v \in V(H)$ such that $\operatorname{deg}_{G}(v)=n-1$. Then $H$ is a connected component of $G$. 
- Let $G$ be a closed distance magic graph and $v$ a vertex of $G$ with $\operatorname{deg} v \leq 1$. Then $v$ lies in a $K_{1}$ or $K_{2}$ component of $G$. In particular, $K_{1}$ and $K_{2}$ are the only trees which are closed distance magic graphs.

- If a graph $G$ has a path wxyz of length 3 , where $\operatorname{deg} x=\operatorname{deg} y=2$, then $G$ is not a closed distance magic graph. In particular, if $C_{n}$ is a component of a closed distance magic graph, then $n \leq 3$.

Corollary 3.10. [1], [9] The cycle $C_{n}$ is not a closed distance magic graph for all $n \geq 4$.

Theorem 3.11. [1] Consider circulant graph $G=C_{n}(c, 2 c, \ldots, k c)$. The graph $G$ is closed distance magic if and only if either $n=2 k c$ or $n=(2 k+1) c$ and $c$ is odd.

Corollary 3.12. [1] Given $n \geq 2$ and $c \geq 1$, the union of $c$ copies of the complete graph, denoted $c K_{n}$, is closed distance magic if and only if $n(c+1) \equiv 0(\bmod 2)$.

Theorem 3.13. [9] The complete partite graph $K_{n_{1}, n_{2}, \ldots, n_{j}}$ is a closed distance magic graph if and only if $n_{i}=1$ for all $i=\{1,2, \ldots, j\}$.

Proposition 3.14. [1] For any $m$ and $n$ such that $2 \leq m \leq n, K_{m, n}$ is not closed distance magic.

Theorem 3.15. [9] Let $G$ be any connected $r$-regular graph, $r>0$. Then $G \otimes K_{n}$, where $n$ is even, is a connected closed distance magic graph.

Corollary 3.16. [1] If the product $G \otimes C_{n}$ is closed distance magic, where $G$ is a regular graph, then $n \equiv 0(\bmod 3)$ or $-1 \in S p(G)$.

Corollary 3.17. [1] Let the product $G \otimes C_{n}$ be closed distance magic, where $G$ is a $2 d$-regular graph on $m$ vertices. Then $n \equiv 1(\bmod 2)$ and $m \equiv 1(\bmod 2)$. Moreover, $n \equiv 3(\bmod 6)$ or $-1 \in S p(G)$.

Theorem 3.18. [1] The strong product $C_{m} \otimes C_{n}$ is closed distance magic if and only if at least one of the following conditions holds:

- $m \equiv 3(\bmod 6)$ and $n \equiv 3(\bmod 6)$

- $\{m, n\}=\{3, x\}$ and $x$ is an odd number.

Theorem 3.19. [1] If $m \equiv 3(\bmod 6)$ and $n \equiv 3(\bmod 6)$, then $C_{m} \otimes C_{n}$ is a closed distance magic graph.

Proposition 3.20. [1] If a strongly regular graph $G$ on $n$ vertices is closed distance magic, then $G \cong K_{n}$.

A regular graph is called strongly regular if there exist constants $a \geq 0, b \geq 1$ such that every pair of adjacent vertices has $a$ common neighbors and every pair of non-adjacent vertices has $b$ common neighbors. 
Theorem 3.21. [1] Let $n$ be an odd number and $r>0$. If $G$ is an r-regular graph of odd order, then $K_{n} \otimes G$ is a closed distance magic graph.

Corollary 3.22. [9] The join graph $C_{n}+H$ is not a closed distance magic graph for any graph $H$. In particular, the wheel $W_{n}=C_{n}+K_{1}$ is not a closed distance magic graph for any value of $n$.

Observation 3.23. [1] Let $C_{m}$ be the cycle $(1,2, \ldots, m)$ and let $A_{j}$ be a set of $p_{j}$ vertices for $j=\{1,2, \ldots, m\}$. We construct a graph $G$ on the vertex set

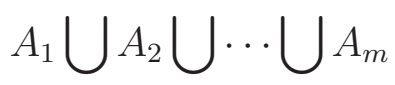

by placing a complete graph $K_{p_{j}}$ on each $A_{j}, j=\{1,2, \ldots, m\}$ and joining each vertex in $A_{i}$ to each each vertex in $A_{j}$, where $\{i, j\}$ is an edge of $C_{m}$. If $m \neq 0(\bmod 3)$, then $G$ is a closed distance magic graph and the sum of the labels in $A_{j}$ is $\frac{\left(\begin{array}{c}n+1 \\ 2\end{array}\right)}{m}$ for $j=\{1,2, \ldots, m\}$.

Notice that if $\left|A_{j}\right|=p$ for all $j \in\{1,2, \ldots, m\}$, then $G=C_{m} \otimes K_{p}$. Observation 3.23 partially provides the motivation for Problem 6.37 in Chapter 6.

Theorem 3.24. [9] Every graph $H$ is an induced subgraph of some regular closed distance magic graph.

Let $V(G)=\left\{x_{1}, x_{2}, \ldots, x_{n}\right\}$. The closed neighborhood adjacency matrix is defined as $A(G)+I_{n}$, where $A(G)$ is the adjacency matrix of $G$ and $I_{n}$ is the $n \times n$ identity matrix.

Corollary 3.25. [41] Let $G$ be any regular graph. If $\left(A(G)+I_{n}\right)^{-1}$ exists, $G$ is not closed distance magic.

Theorem 3.26. [35] Let $G$ be a closed distance magic labeled graph with labeling $\ell$ and magic constant $k^{\prime}$. Then $\sum_{y \in V(G)} \ell(y)(\operatorname{deg}(y)+1)=n k^{\prime}$.

Theorem 3.27. [35] Let $G$ be a closed distance magic labeled graph with labeling $\ell$ and magic constant $k^{\prime}$. Then $\sum_{y \in V(G)} \ell(y)(\operatorname{deg}(y)-1)=n\left(k^{\prime}-(n+1)\right)$.

Theorem 3.28. [35] Let $G$ be a closed distance magic graph on $n$ vertices. Then the magic constant $k^{\prime}=\frac{n(n-1)}{2}$ if and only if $G$ has a vertex of degree $n-1$ in which case $n \equiv 1(\bmod 2)$.

Theorem 3.29. [35] A graph $G$ on $n$ vertices is a closed distance magic graph with magic constant $k^{\prime}=\frac{(n-2)(n+1)}{2}$ if and only if $G$ is $(n-2)$-regular.

Theorem 3.30. [35] A closed distance magic graph on $n$ vertices with magic constant $k^{\prime}=\frac{n(n+1)}{2}-(n+2)$ does not exist. 
Let $\operatorname{Sp}(G)$ denote the spectrum of $G$. The spectrum of a graph $G$ is the set of numbers which are eigenvalues of $A(G)$ together with their multiplicities as eigenvalues of $A(G)$. [10]

Theorem 3.31. [1] Consider the system $w\left(x_{1}\right)=k^{\prime}, w\left(x_{2}\right)=k^{\prime}, \ldots, w\left(x_{n}\right)=k^{\prime}$. This is equivalent to writing $\left(A(G)+I_{n}\right) \ell=k^{\prime} \boldsymbol{u}_{n}$, where $\ell=\left(\ell\left(x_{1}\right), \ldots, \ell\left(x_{n}\right)\right)$ and $\boldsymbol{u}_{n}$ is the all ones vector of length $n$. If $G$ is a closed distance magic graph and the above system has $m+1$ linearly independent solutions, then the multiplicity of -1 in $\operatorname{Sp}(G)$ is $\mathrm{m}$.

Corollary 3.32. [1] Let $G$ be a closed distance magic graph such that there exist $m$ linearly independent solutions to the system $\left(A(G)+I_{n}\right) \ell=k^{\prime} \boldsymbol{u}_{n}$ such that no bijection $\ell: V(G) \rightarrow\{1, \ldots,|V(G)|\}$ is their linear combination. Then the multiplicity of -1 in $S p(G)$ is at least $m$.

Corollary 3.33. [1] If $G$ is a regular closed distance magic graph, then $-1 \in S p(G)$.

A perfect code is a subset $C(G)$ of $V(G)$ such that the closed neighborhoods of the vertices $x \in C$ form the partition of $G$.

Corollary 3.34. [1] Let $G$ be a regular closed distance magic graph having a perfect code. Then the multiplicity of $-1 \in S p(G)$ is at least 2.

Corollary 3.35. [1] If graphs $G$ and $H$ are regular and $G \otimes H$ is closed distance magic, then $-1 \in S p(G) \cup S p(H)$.

The line graph of $G$, denoted $L(G)$, has as its vertices the edges of $G$ and two edges in $L(G)$ are adjacent if they have an end in common. [11]

Corollary 3.36. [1] Let $G$ be an $r$-regular graph, $r>1$. If its line graph $L(G)$ is closed distance magic, then $1-r \in S p(G)$.

Proposition 3.37. [1] For given $n$ and $k, 1<k \leq\left\lfloor\frac{n-3}{2}\right\rfloor$, let the multiplicity of $-1 \in S p(C(n,\{1, \ldots, k-1, k+1\}))$ be $m$. Then $m=m_{1}+m_{2}$, where $m_{1}=2 \mid\{t \mid 1 \leq$ $t \leq k, n t \equiv 0(\bmod 2 k+1)\} \mid$ and $m_{2}=2$ if $n \equiv 0(\bmod 6)$ and $m_{2}=0$ otherwise. In particular, if the graph $C(n,\{1, \ldots, k-1, k+1\})$ is closed distance magic, then $n t \equiv 0(\bmod 2 k+1)$ for some $t \in\{1, \ldots, k\}$ or $n \equiv 0(\bmod 6)$.

\subsubsection{Distances Greater than 1}

A bijection $\ell: V \rightarrow\{1,2, \ldots, n\}$ is said to be a $D$-distance magic labeling if there exists a $D$-distance magic constant $k^{\prime}$ such that for any vertex $x$,

$$
w^{\prime}(x)=\sum_{y \in N_{G}^{D}(x)} \ell(y)=k^{\prime},
$$


where $N_{G}^{D}(x)=\{y \in V \mid d(x, y) \in D\}$. A graph admitting a $D$-distance magic labeling is called $D$-distance magic. Certainly a $\{1\}$-distance magic labeling is a distance magic labeling and a $\{0,1\}$-labeling is a closed distance magic labeling. [53]

We define $d$ as the diameter of graph $G$; that is, the greatest distance between two vertices of $G$. [11] Because the only $\{0\}$-distance magic graph is the trivial graph, we exclude it from consideration. Graph $G$ is defined as $(D, r)$-regular if for all $x \in V(G),\left|N_{G}^{D}(x)\right|=r$; that is, all $D$-neighborhoods have the same cardinality. [53]

We define the set subtraction of $A$ and $B$, denoted $A-B$, as $A-B=\{x \mid x \in$ $A$, and $x \notin B\}$.

Lemma 3.38. [41] Let $D \subset\{0,1, \ldots, d\}$ and $D^{*}=\{0,1, \ldots, d\}-D$. Then $G$ is $D$-distance magic if and only if $G$ is $D^{*}$-distance magic.

Let $V(G)=\left\{x_{1}, x_{2}, \ldots, x_{n}\right\}$. The distance $D$ adjacency matrix, denoted $A_{D}=$ $\left[a_{i, j}\right]$, is defined as the $n \times n$ binary matrix with $a_{i, j}=1$ if and only if $d\left(x_{i}, x_{j}\right) \in D$.

Theorem 3.39. [41] Let $D \subset\{0,1, \ldots, d\}$. If $G$ is $(D, r)$-regular and $A_{D}^{-1}$ exists, then $G$ is not D-distance magic.

For example, consider the Petersen graph $P$ in Figure 3.1, which is 3-regular with order 10 and diameter 2. Let $A$ be the adjacency matrix for $P$. Because $\operatorname{det}(A)=48 \neq 0, P$ is not distance magic by Corollary 2.76. By Lemma 3.38, $P$ is not $\{0,2\}$-distance magic. Let $A+I$ be the closed neighborhood matrix for $P$. Because $\operatorname{det}(A+I)=128 \neq 0, P$ is not closed distance magic by Theorem 3.39. By Lemma $3.38, P$ is also not $\{2\}$-distance magic. [41]
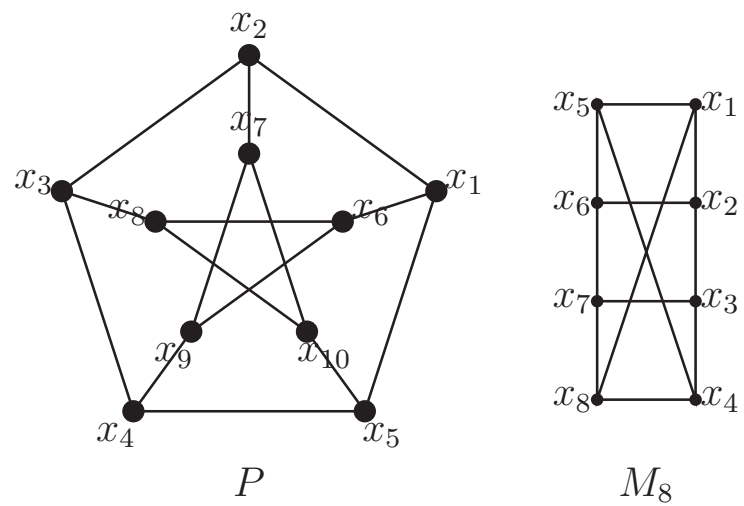

Figure 3.1: Petersen Graph and Möbius Ladder on 8 vertices

Proposition 3.40. [41] Let $M_{n}$ be a Möbius Ladder of order $2 n$ with $n>2 . M_{n}$ is neither distance magic nor closed distance magic.

For example, consider $M_{8}$ in Figure 3.1. By Proposition 3.40, $M_{8}$ is neither distance magic nor closed distance magic. Because $M_{8}$ has diameter 2 , by Lemma 3.39 
and the previous statement, we know it is also not $\{0,2\}$-distance magic or $\{2\}$ distance magic. [41]

Lemma 3.41. [53] Simunjuntak et al. established a number of structural constraints for D-distance magic graphs.

- Let $G$ be a graph having connected components $G_{1}, G_{2}, \ldots, G_{m}$, of diameters $d_{1}, d_{2}, \ldots, d_{m}$ respectively. Let $D \subseteq\left\{0,1, \ldots, d_{\max }\right\}$ and $D^{*}=\left\{0,1, \ldots, d_{\max }\right\}-$ $D$, where $d_{\max }=\max d_{i}$. If $G$ admits a D-distance magic labeling $\ell$ such that $\sum_{y \in G_{i}} \ell(y)$ is constant for each $i$, then $G$ is $D^{*}$-distance magic. Conversely, if $G$ admits a $D^{*}$-distance magic labeling $\ell^{*}$ such that $\sum_{y \in G_{i}} \ell^{*}(y)$ is constant for each $i$, then $G$ is $D$-distance magic.

- If a graph $G$ contains two distinct vertices $x$ and $y$ such that $\left|N_{D}(x) \cap N_{G}^{D}(y)\right|=$ $\left|N_{G}^{D}(x)\right|-1=\left|N_{G}^{D}(y)\right|-1$, then $G$ is not D-distance magic.

- A graph of diameter $d$ is not $\{1,2, \ldots, d\}$-distance magic.

- If $G$ contains a vertex $x$ with $N_{G}^{D}(x)=\emptyset$, then $G$ is not D-distance magic.

- If $G$ contains two distinct vertices $x$ and $y$ such that $N_{G}^{D}(x) \subseteq N_{G}^{D}(y)$, then $G$ is not D-distance magic.

- If each vertex in $G$ has a unique vertex at distance $d$, then $G$ is $\{1,2, \ldots, d-1\}$ distance magic.

Lemma 3.42. [53] Every connected graph is $\{0,1, \ldots, d\}$-distance magic.

Because of Lemma 3.42, we call the $\{0,1, \ldots, d\}$-distance magic graph of $G$ the trivial D-distance magic labeling of $G$.

Proof. Suppose $G$ has order $n$. Because $G$ is connected and has diameter $d$, each $x \in G$ is an element of the neighborhood of each vertex so $k^{\prime}=\frac{n(n+1)}{2}$.

Lemma 3.43. [53] Let $G$ be a non-connected graph with connected components $G_{1}, G_{2}, \ldots, G_{m}$, of diameters $d_{1}, d_{2}, \ldots, d_{m}$ respectively. Let $d_{\max }=\max d_{i}$ and $\left|V\left(G_{i}\right)\right|=n$ for each $i . \quad G$ is $\left\{0,1, \ldots, d_{\max }\right\}$-distance magic if and only if $n$ is even or both $n$ and $m$ are odd.

Theorem 3.44. [53]

- Every even cycle $C_{2 m}$ is $\{1,2, \ldots, m-1\}$-distance magic.

- Every even cycle $C_{2 m}$ is $\{0, m\}$-distance magic.

- For $m$ a positive integer, a cycle $C_{n}$ is $\{m\}$-distance magic if and only if $n=4 m$.

- For $m$ a positive integer, a cycle $C_{n}$ is $\left\{0,1, \ldots, m-1, m+1, \ldots,\left\lfloor\frac{n}{2}\right\rfloor\right\}$-distance magic if and only if $n=4 \mathrm{~m}$. 
- For $m$ a positive integer, a 2-regular graph is $\{m\}$-distance magic if and only if it is a disjoint union of $C_{4 m}$ 's.

- For $n \geq 2 m+2$ a cycle $C_{n}$ is not $\{0,1, \ldots, m\}$-distance magic.

- For $n \geq 2 m+2$ a cycle $C_{n}$ is not $\left\{m+1, m+2, \ldots,\left\lfloor\frac{n}{2}\right\rfloor\right\}$-distance magic

Lemma 3.45. [53] Let $D$ be a distance set containing 2 but not 0 . If $G$ is a graph of diameter at least 2 containing either

- two adjacent vertices of degree one, or

- two vertices of distance 2 having the same neighborhood,

then $G$ is not D-distance magic.

Theorem 3.46. [41] Let $G$ be a graph of even order and let $D \subset\{0,1, \ldots, d\}$. If $G$ is $(D, r)$-regular with odd $r$, then $G$ is not D-distance magic.

Corollary 3.47. [41] Let $D \subset\{1,2, \ldots, d\}$. If $G$ is $(D, r)$-regular with odd $r$, then $G$ is not D-distance magic.

Theorem 3.48. [27], [53] An odd order r-regular graph of diameter 2 is $\{0,2\}$ distance magic if and only if $r$ is even and $2 \leq r \leq n-2$.

Theorem 3.49. [53] There exists an infinite family of regular graphs with diameter 3 admitting a $\{1,2\}$-distance magic labeling.

Corollary 3.50. There exists an infinite family of regular graphs with diameter 3 admitting a $\{0,3\}$-distance magic labeling.

Theorem 3.51. [27], [53] Let $G$ be an odd order regular graph of diameter 2 and let $n$ be an odd positive integer. Then the graph $G \circ \bar{K}_{n}$ is $\{0,2\}$-distance magic.

Theorem 3.52. [53] A complete multipartite graph is not $\{2\}$-distance magic.

Theorem 3.53. [40], [53] Let $m, n>1$. The complete $m$ partite graph with each part of size $n$ is $\{0,2\}$-distance magic if and only if either $n$ is even or both $n$ and $m$ are odd.

Theorem 3.54. [40], [53] Let $1 \leq n_{1} \leq n_{2}$. Let $s_{i}=\sum_{j=1}^{i} n_{j}$ for complete bipartite graph $K_{n_{1}, n_{2}}$. There exists a $\{0,2\}$-distance magic labeling for $K_{n_{1}, n_{2}}$ if and only if the following conditions hold:

- $n_{2} \geq 2$,

- $n(n+1) \equiv 0(\bmod 4)$, where $n=s_{2}$, and

- $\sum_{j=1}^{s_{i}}(n+1-j) \geq \frac{i n(n+1)}{4}$ for $i=\{1,2\}$. 
Theorem 3.55. [40], [53] Let $1 \leq n_{1} \leq n_{2} \leq n_{3}$. Let $s_{i}=\sum_{j=1}^{i} n_{j}$ for complete tripartite graph $K_{n_{1}, n_{2}, n_{3}}$. There exists a $\{0,2\}$-distance magic labeling for $K_{n_{1}, n_{2}, n_{3}}$ if and only if the following conditions hold:

- $n_{2} \geq 2$,

- $n(n+1) \equiv 0(\bmod 6)$, where $n=s_{3}$, and

- $\sum_{j=1}^{s_{i}}(n+1-j) \geq \frac{i n(n+1)}{6}$ for $i=\{1,2,3\}$.

Theorem 3.56. [52] For $n \geq 1, C_{4} \circ \bar{K}_{n}$ is $\{0,2\}$-distance magic.

We also can calculate the magic constant for a distance magic graph by using dominating functions. A function $g: V(G) \rightarrow \mathbb{R}^{+}=[0, \infty)$ is said to be a $D$-neighborhood fractional dominating function if for every vertex $v \in V(G), \sum_{u \in N_{D}(v)} g(u) \geq 1$. The $D$-neighborhood fractional domination number of $G$, denoted $\gamma_{f}(G ; D)$ is defined as $\gamma_{f}(G ; D)=\min \left\{\sum_{v \in V(G)} g(v) \mid g\right.$ is a $D$-neighborhood fractional dominating function. $\}$

Theorem 3.57. [44] If graph $G$ is D-distance magic, then its D-distance magic constant $k=\frac{n(n+1)}{2 \gamma_{f}(G ; D)}$.

For more information on fractional dominating functions and how close certain graphs are to being distance magic or D-distance magic, see [41], [42], [44].

In [53], Simanjuntak et al. asked,

Problem 3.58. Does there exist a graph of diameter at least 3 admitting a $\{0,1,2\}$ distance magic labeling?

The answer is yes. By Lemma 3.38, a graph of diameter 3 with a $\{3\}$-distance magic labeling also has a $\{0,1,2\}$-distance magic labeling. We define the bowtie product, denoted $G \bowtie H$ as a graph with vertex set $V(G) \times V(H)$. Vertices $(g, h)$ and $\left(g^{\prime}, h^{\prime}\right)$ in $G \bowtie H$ are adjacent if and only if $g$ is adjacent to $g^{\prime}$ and $h$ is adjacent to $h^{\prime}$ or $g=g^{\prime}$ and $h$ is adjacent to $h^{\prime}$. Consider $C_{3} \bowtie C_{7}$ in Figure 3.2. Each vertex has 2 vertices of distance 3 in each column. The magic constant for the $\{0,1,2\}$-labeling is 165 .

\subsection{Distance Antimagic Graphs}

A concept related to magic graphs is that of antimagic graphs. A distance d-antimagic labeling of a graph $G=G(V, E)$ is a bijection $f: V(G) \rightarrow\{1,2, \ldots, n\}$ with the property that there exists an ordering of the vertices of $G$ such that the sequence of the weights $w\left(x_{1}\right), w\left(x_{2}\right), \ldots, w\left(x_{n}\right)$ forms an arithmetic progression with difference $d$. When $d=1, f$ is just called a distance antimagic labeling. A graph $G$ is a distance $d$ antimagic graph if it allows a distance $d$-antimagic labeling, and a distance antimagic graph when $d=1$. [23] 


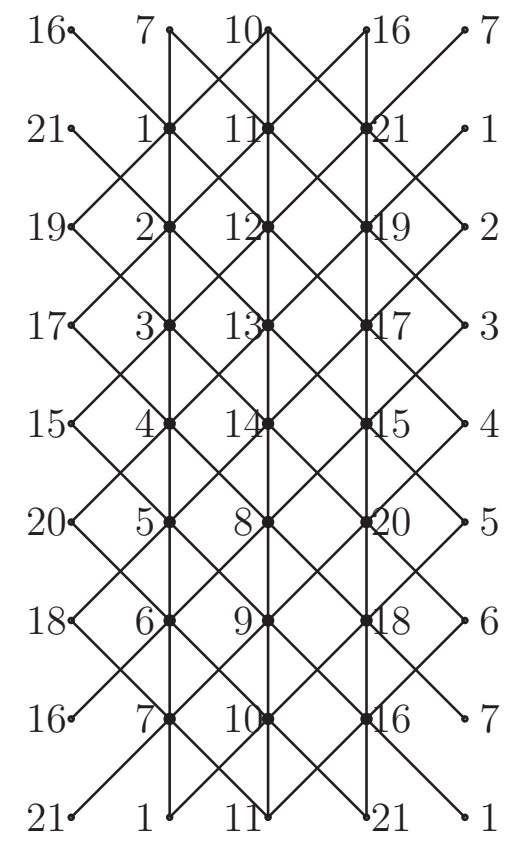

Figure 3.2: $\{3\}$-Distance Labeled Graph

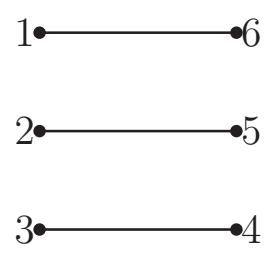

Figure 3.3: Perfect Matching on 6 Vertices

Observation 3.59. [23] If a graph $G$ is distance magic, then its complement $\bar{G}$ is distance antimagic.

An ordered distance antimagic labeling of a graph $G(V, E)$ with $n$ vertices is a bijection $\vec{f}: V \rightarrow\{1,2, \ldots, n\}$ with the property that $\vec{f}\left(v_{i}\right)=i$ and the sequence of the weights $w\left(x_{1}\right), w\left(x_{2}\right), \ldots, w\left(x_{n}\right)$ forms an increasing arithmetic progression with difference one. A graph $G$ is an ordered distance antimagic graph if it allows an ordered distance antimagic labeling.

For example, consider the perfect matching on 6 vertices, which is the complement of the 4-regular graph on 6 vertices. Clearly this graph admits an ordered distance antimagic labeling as shown in Figure 3.3.

Theorem 3.60. [24] Let $a, b$ be positive integers such that $a, b>1, a b>4$, and $a \equiv b(\bmod 2)$. Let $n=a b$ and $r=n-a-b+1$. Then there exists an $r$-regular ordered distance antimagic graph with $n$ vertices. 
Theorem 3.61. [24] The graph $G=K_{a} \square K_{b}$ is distance 2-antimagic when $a, b>1$, $a b>4$, and $a \equiv b(\bmod 2)$.

A magic rectangle set $\mathcal{M}=M R S(a, b ; c)$ is a collection of $c$ arrays $(a \times b)$ whose entries are elements of $\{1,2, \ldots, a b c\}$, each appearing once, with all row sums in every rectangle equal to a constant $s$ and all column sums in every direction equal to a constant $t$.

Observation 3.62. [23] The graph $G=c\left(K_{a} \square K_{b}\right)$ admits a distance 2-antimagic labeling $f$ such that $f(x)=m$ implies $w_{G}(x)=\frac{(a+b)(a b c+1)}{2}-2 m$ for every $x \in V(G)$ whenever there exists a magic rectangle set $M R S(a, b ; c)$.

For more details on magic rectangle sets, see [23], [25]. 


\section{Chapter 4}

\section{Different Labels}

Up to this point, all of our labelings have been bijections from $V(G) \rightarrow\{1,2, \ldots, n\}$. However, we can consider other sets of labels and determine whether they have a magic constant.

\subsection{Group Distance Magic Graphs}

A more common extension of distance magic graphs is to expand the label set to an Abelian group of order $n$. We use the following definition. A graph $G=G(V, E)$ of order $n$ has a group distance magic labeling (or $\Gamma$-distance magic labeling ) if there is an injection $\ell$ from $V$ to an Abelian group $\Gamma$ of order $n$ such that the weight $w(x)=\sum_{x \in N_{G}(x)} \ell(x)=\mu$ for all $x \in V$ where $\mu \in \Gamma$ is called the magic constant. [22]

\subsection{1 $\mathbb{Z}_{n}$-Distance Magic Graphs}

We begin with some theorems on $\mathbb{Z}_{n}$, because these groups provide labelings that are the closest to behaving like distance magic labelings.

Observation 4.1. [22] Every graph with $n$ vertices and a distance magic labeling also admits a $\mathbb{Z}_{n}$-distance magic labeling, but the converse need not be true.

For example, the graph in Figure $4.1(\mathrm{a})$ is a $\mathbb{Z}_{6}$-distance magic labeling of a 4 regular graph on 6 vertices with magic constant $\mu=10$. Note the similarity to the distance magic graph in the introduction. We contrast this with the graph in Figure 4.1(b). By Theorem 2.81, we know $C_{3} \square C_{4}$ has no distance magic labeling. However, a $\mathbb{Z}_{12}$-distance magic labeling of $C_{3} \square C_{4}$ with magic constant $\mu=0$ exists as is shown in Figure 4.1(b).

Theorem 4.2. [17] If $G$ is an $r$-regular distance magic graph on $n$ vertices, where $r$ is odd then $G$ is not $\mathbb{Z}_{n}$-distance magic.

Corollary 4.3. [17] If $\operatorname{gcd}(n, d+1)=2 k+1, p$ is even, $n=2 m(2 k+1)$ and $\operatorname{gcd}(2 k+1,2 m)=1$, then $C_{n}(1, d)$ has a $\mathbb{Z}_{n}$-magic labeling. 


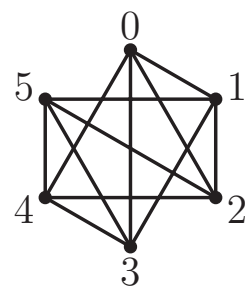

(a)

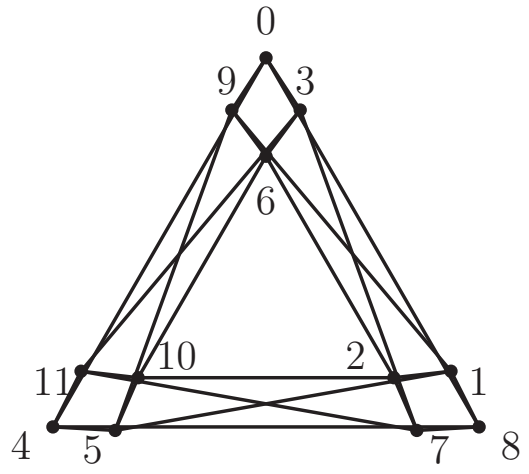

(b)

Figure 4.1: (a) $\mathrm{A} \mathbb{Z}_{6}$-Distance Magic graph and (b) a $\mathbb{Z}_{12}$-Distance Magic Graph

Corollary 4.4. [17] If $n=m\left(d^{2}-1\right)$ and

- $m=1$ if $d$ is odd

- $m=2 h$ and $\operatorname{gcd}(d+1,2 h)=1$ if $d$ is even

then $C_{n}(1, d)$ is $\mathbb{Z}_{n}$-distance magic.

Corollary 4.5. [12] Let $m$ and $n$ be two positive integers such that $m$ is odd and $n$ is even. The graph $K_{m, n}$ is not a $\mathbb{Z}_{4 m+4 n}$-distance magic graph.

Observation 4.6. [15] Let $G=K_{n_{0}, n_{1}, n_{2}, \ldots, n_{m-1}}$ be a complete m-partite graph such that $1 \leq n_{0} \leq n_{1} \leq \cdots \leq n_{m-1}$ and $n=n_{0}+n_{1}+\cdots+n_{m-1}$ is odd. $G$ is a $\mathbb{Z}_{n}$-distance magic graph if and only if $n_{i} \geq 2$ for $i=1,2, \ldots, m-1$.

Theorem 4.7. [3] A graph $C_{m} \times C_{n}$ is $\mathbb{Z}_{m n}$-distance magic if and only if $m \in\{4,8\}$ or $n \in\{4,8\}$ or $m, n \equiv 0(\bmod 4)$.

Theorem 4.8. [22] The Cartesian product $C_{m} \square C_{n}, m, n \geq 3$ is a $\mathbb{Z}_{m n}$-distance magic graph if and only if $m n$ is even.

Observation 4.9. [22] When both $m$ and $n$ are even, then there exists a $\mathbb{Z}_{m n^{-}}$ distance magic labeling of $C_{m} \square C_{n}$ for any magic constant $\mu \in \mathbb{Z}_{m n}, \mu \equiv 2(\bmod 4)$. When $m \equiv 0(\bmod 4)$ and $n$ is odd, then there exists a $\mathbb{Z}_{m n}$-distance magic labeling of $C_{m} \square C_{n}$ for any magic constant $\mu \in \mathbb{Z}_{m n}, \mu \equiv 0(\bmod 4)$. When $m \equiv 2(\bmod 4)$ and $n$ is odd, then there exists a $\mathbb{Z}_{m n}$-distance magic labeling of $C_{m} \square C_{n}$ for any magic constant $\mu \in \mathbb{Z}_{m n}, \mu \equiv 0(\bmod 2)$.

A $\mathbb{Z}_{n}$-balanced labeling of an $r$-regular graph $G$ with $n$ vertices is a bijection $\ell: V(G) \rightarrow \mathbb{Z}_{n}$ such that $w(x) \equiv r \ell(x)(\bmod n)$ for every $x \in V(G)$.

Lemma 4.10. [19] 
- The cycle $C_{n}$ for any $n \geq 3$ admits a $\mathbb{Z}_{n}$-balanced labeling.

- The complete graph $K_{n}$ on $n \geq 2$ vertices admits a $\mathbb{Z}_{n}$-balanced labeling if and only if $n$ is odd.

Lemma 4.11. [19] Let $G_{1}$ and $G_{2}$ be regular graphs. If both $G_{1}$ and $G_{2}$ have $\mathbb{Z}_{n^{-}}$ balanced labelings, then $G_{1} \otimes G_{2}$ has a $\mathbb{Z}_{n}$-balanced labeling.

\subsubsection{General $\Gamma$-Distance Magic Graphs}

Certainly many other group structures exist besides $\mathbb{Z}_{n}$. The following theorems concern labelings from any Abelian group.

Theorem 4.12. [15] The complete bipartite graph $K_{m, n}$ is a group distance magic graph if and only if $m+n \neq \equiv 2(\bmod 4)$.

Theorem 4.13. [15] Let $G=K_{n_{0}, n_{1}, n_{2}, \ldots, n_{m-1}}$ be a complete m-partite graph and $n=n_{0}+n_{1}+\cdots+n_{m-1}$. If $n \equiv 2(\bmod 4)$ and $m$ is even, then there does not exist an Abelian group $\Gamma$ of order $n$ such that $G$ is a $\Gamma$-distance magic graph.

The exponent of a finite Abelian group $\Gamma$, denoted $\exp (\Gamma)$, is the least positive integer $m$ such that $m x=0$ for every $x \in \Gamma$ [49]. For example, $\exp \left(\mathbb{Z}_{n_{1}} \times \cdots \times \mathbb{Z}_{n_{j}}\right)=$ $\operatorname{lcm}\left(n_{1}, \ldots, n_{j}\right)$.

Given Abelian group $\Gamma$ and a subset $S \subseteq \Gamma \backslash\{0\}$ such that $-s \in S$ whenever $s \in S$, the Cayley graph $\operatorname{Cay}(\Gamma ; S)$ of $\Gamma$ with respect to connection set $S$ has vertex set $\Gamma$ such that $x, y \in \Gamma$ are adjacent if and only if $x-y \in S(\operatorname{so} \operatorname{Cay}(\Gamma ; S)$ is an $|S|$-regular graph). [19]

Theorem 4.14. [19] Let $\Gamma$ be a finite Abelian group of order $n=|\Gamma|$, and $G=$ $C a y(\Gamma, S)$, a Cayley graph on $\Gamma$ of degree $r=|S|$. If $\exp (\Gamma)$ is a divisor of $r$, then $G$ is $\Gamma$-distance magic, and any automorphism $f$ of $\Gamma$ is an $\Gamma$-distance magic labeling of $G$ with magic constant $\mu=\sum_{s \in S} f(s)$.

Corollary 4.15. [19] Let $p \geq 2$ be a prime, and let $r \geq 2$ and $d \geq 1$ be integers. If $p$ is a divisor of $r$, then any Cayley graph $C a y\left(\mathbb{Z}_{p}^{d} ; S\right)$ on $\mathbb{Z}_{p}^{d}$ with degree $r$ is $\mathbb{Z}_{p^{-}}^{d}$ distance magic and any automorphism $f$ of $\mathbb{Z}_{p}^{d}$ is a $\mathbb{Z}_{p}^{d}$-distance magic labeling with magic constant $\mu=\sum_{s \in S} f(s)$.

Corollary 4.16. [19] Let $d \geq 2$ be an integer. Any Cayley graph Cay $\left(\mathbb{Z}_{2}^{d} ; S\right)$ on $\mathbb{Z}_{2}^{d}$ with an even degree $|S|$ is $\mathbb{Z}_{2}^{d}$-distance magic with any automorphism $f$ of $\mathbb{Z}_{2}^{d}$ as a $\mathbb{Z}_{2}^{d}$-distance magic labeling with magic constant $\mu=\sum_{s \in S} f(s)$.

Let $Q_{n}$ be the $n$-dimensional hypercube where vertices are adjacent if and only if the labels have Hamming distance one; that is, $Q_{n}$ is the graph with binary strings of length $n$ as its vertices and two vertices are adjacent if and only if the corresponding 
strings differ in exactly one position. Equivalently, the $n$-dimensional hypercube $Q_{n}$ is the Cayley graph $\operatorname{Cay}\left(\mathbb{Z}_{2}^{n} ; S\right)$ with

$$
S=\{(1,0, \ldots, 0),(0,1, \ldots, 0), \ldots,(0,0, \ldots, 1)\} .
$$

Corollary 4.17. [18] Let $n \geq 2$ be an integer. If $n$ is even, then $Q_{n}$ is $\mathbb{Z}_{2}^{n}$-distance magic with magic constant $(1, \ldots, 1)$.

Theorem 4.18. [22] The graph $C_{2^{m}} \times C_{2^{m}}$ has a $\mathbb{Z}_{2}^{2 m}$-distance magic labeling for $m \geq 2$ and the magic constant $\mu=(0,0, \ldots, 0)$.

Let $\oplus$ denote addition in $F_{2}^{n}$ and let $e_{1}, \ldots, e_{n}$ be the standard basis of $\mathbb{F}_{2}^{n}$. We say a set $A$ is balanced if $\left|A_{i}^{0}\right|=\left|A_{i}^{1}\right|$ for every $i \in[n]=\{1, \ldots, n\}$. A bijection $f: \mathbb{F}_{2}^{n} \rightarrow$ $\mathbb{F}_{2}^{n}$ is neighbor-balanced if the set $L(x)=\left\{f(y) \mid y \in N_{Q_{n}}(x)\right\}=\left\{f\left(x \oplus e_{i}\right) \mid i \in[n]\right\}$ is balanced for every $x \in \mathbb{F}_{2}^{n}$.

Proposition 4.19. [30] Every neighbor-balanced $f: \mathbb{F}_{2}^{n} \rightarrow \mathbb{F}_{2}^{n}$ is a $\mathbb{Z}_{2}^{n}$-distance magic labeling of $Q_{n}$.

Theorem 4.20. [30] A neighbor-balanced $f: \mathbb{F}_{2}^{n} \rightarrow \mathbb{F}_{2}^{n}$ exists for every $n \equiv 2$ $(\bmod 4)$.

For example, consider matrix $M$ over $\mathbb{F}_{2}$ such that

$$
M=\left[\begin{array}{llllll|ll|llllll}
1 & 0 & 0 & 0 & 0 & 0 & 0 & 0 & 1 & 1 & 1 & 1 & 1 & 1 \\
0 & 1 & 0 & 0 & 0 & 0 & 0 & 0 & 1 & 1 & 1 & 1 & 1 & 1 \\
0 & 0 & 1 & 0 & 0 & 0 & 0 & 0 & 1 & 1 & 1 & 1 & 1 & 1 \\
0 & 0 & 0 & 1 & 0 & 0 & 0 & 0 & 1 & 1 & 1 & 1 & 1 & 1 \\
0 & 0 & 0 & 0 & 1 & 0 & 0 & 0 & 1 & 1 & 1 & 1 & 1 & 1 \\
0 & 0 & 0 & 0 & 0 & 1 & 0 & 0 & 1 & 1 & 1 & 1 & 1 & 1 \\
\hline 0 & 0 & 0 & 0 & 0 & 0 & 1 & 0 & 1 & 1 & 1 & 1 & 1 & 1 \\
0 & 0 & 0 & 0 & 0 & 0 & 0 & 1 & 1 & 1 & 1 & 1 & 1 & 1 \\
\hline 1 & 1 & 1 & 1 & 1 & 1 & 0 & 0 & 1 & 0 & 0 & 0 & 0 & 0 \\
1 & 1 & 1 & 1 & 1 & 1 & 0 & 0 & 0 & 1 & 0 & 0 & 0 & 0 \\
1 & 1 & 1 & 1 & 1 & 1 & 0 & 0 & 0 & 0 & 1 & 0 & 0 & 0 \\
1 & 1 & 1 & 1 & 1 & 1 & 0 & 0 & 0 & 0 & 0 & 1 & 0 & 0 \\
1 & 1 & 1 & 1 & 1 & 1 & 0 & 0 & 0 & 0 & 0 & 0 & 1 & 0 \\
1 & 1 & 1 & 1 & 1 & 1 & 0 & 0 & 0 & 0 & 0 & 0 & 0 & 1
\end{array}\right] .
$$

Matrix $M$ has columns that form a balanced set and is invertible. Thus consider the map $f(x)=M x^{T}$ for $x \in \mathbb{F}_{2}^{6}=\mathbb{Z}_{2}^{6}$. Now, the vertices of $Q_{6}$ are the elements of $\mathbb{Z}_{2}^{6}$ so vertex $x$ being adjacent to $y$ is equivalent to having the distance between vertices $x$ and $y$ equal to 1 . The label on vertex $x$ is $M x^{T}$.

The size of a vector $x \in \mathbb{F}_{2}^{n}$ is the number of ones in $x$. For $1 \leq d \leq n$, let $D_{d}$ denote the $n \times\left(\begin{array}{l}n \\ d\end{array}\right)$ matrix over $\mathbb{F}_{2}^{n}$ whose columns are precisely all vectors of size $d$ (in some fixed linear order of $\mathbb{F}_{2}^{n}$ ). [30] 
Proposition 4.21. [30] Every $D_{d}$-balanced $f: \mathbb{F}_{2}^{n} \rightarrow \mathbb{F}_{2}^{n}$ is a d-distance magic labeling of $Q_{n}$.

An $n \times k$ matrix over $\mathbb{F}_{2}^{n}$ is balanced if the set of its columns is balanced; that is, it has the same number of ones and zeros in each row. [30]

Lemma 4.22. [30] If $A$ is a balanced $n \times n$ matrix, then $A D_{d}$ is balanced for every odd $1 \leq d \leq n$.

Theorem 4.23. [30] For every $n \equiv 2(\bmod 4)$ there exists $f: \mathbb{F}_{2}^{n} \rightarrow \mathbb{F}_{2}^{n}$ that is $D_{d}$-balanced for every odd $1 \leq d \leq n$.

Corollary 4.24. [30] For every $n \equiv 2(\bmod 4)$ there is a $\mathbb{Z}_{2}^{n}$-distance magic labeling of $Q_{n}$ that is group $d$-distance magic for every odd $1 \leq d \leq n$.

Let $Q_{n}(S)$ be the graph on $\{0,1\}^{n}$ with edges between two vertices whenever they differ on exactly $s \in S$ coordinates.

Corollary 4.25. [30] $Q_{n}(S)$ has a group distance magic labeling for every $n \equiv 2$ (mod 4$)$ and every $S \subseteq\{1,2, \ldots, n\}$ containing only odd integers.

Notice this is equivalent to stating $Q_{n}(S)$ has a group $D$-distance magic labeling for every $n \equiv 2(\bmod 4)$ and every $D \subseteq\{1,2, \ldots, n\}$ containing only odd integers.

Most of the work with group distance magic graphs involves graph products. Like in chapter 2, we separate these results by the specific graph product.

\section{Lexicographic Product}

Recall that the lexicographic product of $G$ and $H$ is denoted $G \circ H$ with vertex set $V(G) \times V(H)$. $G \circ H$ is created by replacing every vertex of $G$ with a copy of $H$. Thus, vertices $(g, h)$ and $\left(g^{\prime}, h^{\prime}\right)$ in $G \circ H$ are adjacent if and only if either $g$ is adjacent to $g^{\prime}$ in $G$ or $g=g^{\prime}$ and $h$ is adjacent to $h^{\prime}$ in $H$. [32]

Lemma 4.26. [16] Let $G$ be a graph of order $n$ and $\Gamma$ be an arbitrary Abelian group of order $4 n$ such that $\Gamma \cong \mathbb{Z}_{2^{m}} \times \mathcal{A}$ for $m \geq 2$ and some Abelian group $\mathcal{A}$ of order $\frac{n}{2^{m-2}}$. If $\operatorname{deg}(x) \equiv c\left(\bmod 2^{m-1}\right)$ for some constant $c$ and any $x \in V(G)$, then there exists a $\Gamma$-distance magic labeling for the graph $G \circ C_{4}$.

Theorem 4.27. [16] Let $G$ be a graph of order $n$ and $\Gamma$ be an arbitrary Abelian group of order $4 n$ such that $\Gamma \cong \mathbb{Z}_{2} \times \mathbb{Z}_{2} \times \mathcal{A}$ for some Abelian group $\mathcal{A}$ of order $n$. There exists a $\Gamma$-distance magic labeling for the graph $G \circ C_{4}$.

Theorem 4.28. [16] Let $G$ be a graph of order $n$ and $\Gamma$ be an Abelian group of order $4 n$. If $n=2^{m}(2 k+1)$ for some natural numbers $m, k$, and $\operatorname{deg}(x) \equiv c\left(\bmod 2^{m+1}\right)$ for some constant $c$ for any $x \in V(G)$, then there exists a $\Gamma$-distance magic labeling for the graph $G \circ C_{4}$. 
Observation 4.29. [16] Let $G$ be a graph of odd order $n$ and $\Gamma$ be an Abelian group of order $4 n$. If $G$ is an Eulerian graph (i.e. all vertices of the graph $G$ have even degrees), then there exists a $\Gamma$-distance magic labeling for the graph $G \circ C_{4}$.

Observation 4.30. [16] Let $K_{m, n}$ be a complete bipartite graph with $m$ even and $n$ odd and let $\Gamma$ be an Abelian group of order $4(m+n)$. There exists a $\Gamma$-distance magic labeling for the graph $K_{m, n} \circ C_{4}$.

\section{Direct Product}

Recall that the direct product, denoted $G \times H$, is a graph with vertex set $V(G) \times V(H)$. Vertices $(g, h)$ and $\left(g^{\prime}, h^{\prime}\right)$ in $G \times H$ are adjacent if and only if $g$ is adjacent to $g^{\prime}$ in $G$ and $h$ is adjacent to $h^{\prime}$ in $H$. [32]

Theorem 4.31. [19] Let $G_{i}$ be an $r_{i}$-regular graph with $n_{i}$ vertices, $i=\{1, \ldots, m\}$. Suppose $n_{i}$ and $r / r_{i}$ are coprime for $i=\{1, \ldots, m\}$, where $r=r_{1} \ldots r_{m}$. If each $G_{i}$ is $\mathbb{Z}_{n_{i}}$-distance magic, then $G_{1} \times \cdots \times G_{m}$ is $\mathbb{Z}_{n_{1} \ldots n_{m}}$-distance magic.

Theorem 4.32. [3] If $G$ is an r-regular graph of order $n$, then direct product $G \times C_{4}$ is a group distance magic graph. ${ }^{1}$

Theorem 4.33. [12]

- Let $G$ be a graph of order $n$. If $n=2^{m}(2 k+1)$ for some natural numbers $m, k$, and $\operatorname{deg}(x) \equiv c\left(\bmod 2^{m+2}\right)$ for some constant $c$ for any $x \in V(G)$, then there exists a group distance magic labeling for the graph $G \times C_{4}$.

- Let $G=K_{q, s, t}$ be a complete tripartite graph with all partite sets odd. Then $G \times C_{4}$ is a group distance magic graph.

- Let $m$ and $n$ be two positive integers such that $m$ is odd and $n$ is even. The graph $K_{m, n} \times C_{4}$ is not a $\Gamma$-distance magic graph for any group $\Gamma$ of order $4 m+4 n$ having exactly one involution $\iota$.

- Let $m$ and $n$ be two positive integers such that $m$ is odd and $n$ is even. The graph $K_{m, n} \times C_{4}$ is a $\Gamma$-distance magic graph if and only if $\Gamma \cong \mathbb{Z}_{2} \times \mathbb{Z}_{2} \times \mathcal{A}$ for a group $\mathcal{A}$ of order $m+n$.

\section{Theorem 4.34.}

- [12] Let $G$ be a graph of order $n$ and $\Gamma$ be an arbitrary Abelian group of order $4 n$ such that $\Gamma \cong \mathbb{Z}_{2} \times \mathbb{Z}_{2} \times \mathcal{A}$ for some Abelian group $\mathcal{A}$ of order $2 n$. If all vertices of $G$ have even degrees, then there exists a $\Gamma$-distance magic labeling for the graph $G \times C_{8}$.

\footnotetext{
${ }^{1}$ This theorem is not stated but can be inferred.
} 
- [12] Let $G$ be a graph of order $n$. If $n=2^{m}(2 k+1)$ for some natural numbers $m, k$, and $\operatorname{deg}(x) \equiv 2 c\left(\bmod 2^{m+2}\right)$ for some constant $c$ for any $x \in V(G)$, then there exists a group distance magic labeling for the graph $G \times C_{8}$.

- [3] If $G$ is an r-regular graph of order $n$ for some even $r$, then direct product $C_{8} \times G$ is a group distance magic graph.

Observation 4.35. [12] Let $m$ and $n$ be two positive integers such that $m \leq n$. If the graph $K_{m, n} \times C_{8}$ is a distance magic graph, then the following conditions hold:

- $m+n \equiv 0(\bmod 2)$ and

- $m \geq \frac{\sqrt{2(16 n+1)^{2}-1}-1}{16}-n$.

Theorem 4.36. [3] If $m, n \equiv 0(\bmod 4)$ then the direct product $C_{m} \times C_{n}$ is $\mathcal{A} \times \mathcal{B}$ distance magic for any Abelian groups $\mathcal{A}$ and $\mathcal{B}$ of order $m$ and $n$ respectively.

Proposition 4.37. [3] If $m, n \equiv 0(\bmod 4)$ then the direct product $C_{m} \times C_{n}$ is $\mathbb{Z}_{t} \times \mathcal{A}$-distance magic for $m \mid t$ and any Abelian group $\mathcal{A}$ of order $\frac{m n}{t}$.

Theorem 4.38. [3] Assume that $m, n \geq 3$ and $m, n \notin\{4,8\}, m=4 b+d$ and $n=4 a+c$ for some integers $a, b \geq 0$, where $c \in\{0,1,2,3\}$ and $d \in\{1,2,3\}$. If an Abelian group $\Gamma$ of order mn has less than $\max \{2, a-1\}$ involutions, then $C_{m} \times C_{n}$ is not $\Gamma$-distance magic.

Theorem 4.39. [3] If $m, n \not \equiv 0(\bmod 4)$, then $C_{m} \times C_{n}$ is not $\Gamma$-distance magic for any Abelian group $\Gamma$ of order $m$.

Corollary 4.40. [3] Assume that $m, n \geq 3$ and $\{m, n\}=\{4 a, 4 b+c\}$ for some integers $a \geq 3$ and $b \geq 0, c \in\{1,2,3\}$. Then $C_{m} \times C_{n}$ can be $\Gamma$-distance magic only in the following cases:

- $c \in\{1,3\}$ and $\Gamma \cong \mathcal{A} \times\left(\mathbb{Z}_{2}\right)^{t+2}$ for some Abelian group $\mathcal{A}$ of odd order, where $a=2^{t}$,

- $c \in\{1,3\}$ and $\Gamma \cong \mathcal{A} \times \mathbb{Z}_{3} \times\left(\mathbb{Z}_{2}\right)^{t}$ for some Abelian group $\mathcal{A}$ of odd order, where $a=3 \cdot 2^{t-2}$,

- $c \in\{1,3\}$ and $\Gamma \cong \mathcal{A} \times\left(\mathbb{Z}_{2}\right)^{t} \times \mathbb{Z}_{4}$ for some Abelian group $\mathcal{A}$ of odd order, where $a=2^{t}$,

- $c \in\{1,3\}$ and $\Gamma \cong \mathcal{A} \times\left(\mathbb{Z}_{2}\right)^{t-2} \times\left(\mathbb{Z}_{4}\right)^{2}$ for some Abelian group $\mathcal{A}$ of odd order, where $a=2^{t}$,

- $c \in\{1,3\}$ and $\Gamma \cong \mathcal{A} \times\left(\mathbb{Z}_{2}\right)^{t-1} \times \mathbb{Z}_{8}$ for some Abelian group $\mathcal{A}$ of odd order, where $a=2^{t}$,

- $c=2$ and $\Gamma \cong \mathcal{A} \times\left(\mathbb{Z}_{2}\right)^{t+3}$ for some Abelian group $\mathcal{A}$ of odd order, where $a=2^{t}$, 
- $c=2$ and $\Gamma \cong \mathcal{A} \times \mathbb{Z}_{3} \times\left(\mathbb{Z}_{2}\right)^{t+1}$ for some Abelian group $\mathcal{A}$ of odd order, where $a=3 \cdot 2^{t-2}$,

- $c=2$ and $\Gamma \cong \mathcal{A} \times \mathbb{Z}_{3} \times\left(\mathbb{Z}_{2}\right)^{t-1} \times \mathbb{Z}_{4}$ for some Abelian group $\mathcal{A}$ of odd order, where $a=3 \cdot 2^{t-2}$,

- $c=2$ and $\Gamma \cong \mathcal{A} \times \mathbb{Z}_{5} \times\left(\mathbb{Z}_{2}\right)^{t}$ for some Abelian group $\mathcal{A}$ of odd order, where $a=5 \cdot 2^{t-3}$,

- $c=2$ and $\Gamma \cong \mathcal{A} \times \mathbb{Z}_{7} \times\left(\mathbb{Z}_{2}\right)^{t}$ for some Abelian group $\mathcal{A}$ of odd order, where $a=7 \cdot 2^{t-3}$,

- $c=2$ and $\Gamma \cong \mathcal{A} \times\left(\mathbb{Z}_{2}\right)^{t+1} \times \mathbb{Z}_{4}$ for some Abelian group $\mathcal{A}$ of odd order, where $a=2^{t}$,

- $c=2$ and $\Gamma \cong \mathcal{A} \times\left(\mathbb{Z}_{2}\right)^{t-1} \times\left(\mathbb{Z}_{4}\right)^{2}$ for some Abelian group $\mathcal{A}$ of odd order, where $a=2^{t}$,

- $c=2$ and $\Gamma \cong \mathcal{A} \times\left(\mathbb{Z}_{2}\right)^{t} \times \mathbb{Z}_{8}$ for some Abelian group $\mathcal{A}$ of odd order, where $a=2^{t}$,

- $c=2$ and $\Gamma \cong \mathcal{A} \times\left(\mathbb{Z}_{2}\right)^{t-2} \times \mathbb{Z}_{4} \times \mathbb{Z}_{8}$ for some Abelian group $\mathcal{A}$ of odd order, where $a=2^{t}$,

- $c=2$ and $\Gamma \cong \mathcal{A} \times\left(\mathbb{Z}_{2}\right)^{t-1} \times \mathbb{Z}_{16}$ for some Abelian group $\mathcal{A}$ of odd order, where $a=2^{t}$.

Observation 4.41. [3] If an $r_{1}$-regular graph $G_{1}$ is $\Gamma_{1}$-distance magic and an $r_{2}$ regular graph $G_{2}$ is $\Gamma_{2}$-distance magic, then the direct product $G_{1} \times G_{2}$ is $\Gamma_{1} \times \Gamma_{2^{-}}$distance magic.

Theorem 4.42. [3] If $G$ is a balanced distance magic graph and $H$ is an r-regular graph for $r \geq 1$, then $G \times H$ is a group distance magic graph.

Proposition 4.43. [3] If $G$ is an r-regular graph of order $n$ for some even $r$, then the direct product $C_{2^{t}} \times G, t \geq 2$, admits an $\mathcal{A} \times \mathcal{B}$-distance magic labeling for any Abelian group $\mathcal{B}$ of order $n$ and an Abelian group $\mathcal{A}$ such that:

- $\mathcal{A} \cong\left(\mathbb{Z}_{2}\right)^{t}$,

- $\mathcal{A} \cong \mathbb{Z}_{4} \times\left(\mathbb{Z}_{2}\right)^{t-2}$,

- $\mathcal{A} \cong \mathbb{Z}_{8} \times\left(\mathbb{Z}_{2}\right)^{t-3}$,

- $\mathcal{A} \cong\left(\mathbb{Z}_{4}\right)^{2} \times\left(\mathbb{Z}_{2}\right)^{t-4}$

Proposition 4.44. [3] If $G$ is an r-regular graph of order $n$ for some even $r$ and $n$, then the direct product $C_{2^{t}} \times G, t \geq 2$, admits an $\mathcal{A} \times \mathcal{B}$-distance magic labeling for any Abelian group $\mathcal{B}$ of order $\frac{n}{2}$ and an Abelian group $\mathcal{A}$ such that: 
- $\mathcal{A} \cong \mathbb{Z}_{8} \times \mathbb{Z}_{4} \times\left(\mathbb{Z}_{2}\right)^{t-4}$

- $\mathcal{A} \cong \mathbb{Z}_{16} \times\left(\mathbb{Z}_{2}\right)^{t-3}$.

\section{Cartesian Product}

Recall that the Cartesian product, denoted $G \square H$, is a graph with vertex set $V(G) \times$ $V(H)$. Vertices $(g, h)$ and $\left(g^{\prime}, h^{\prime}\right)$ in $G \square H$ are adjacent if and only if $g=g^{\prime}$ and $h$ is adjacent to $h^{\prime}$ in $H$, or $h=h^{\prime}$ and $g$ is adjacent to $g^{\prime}$ in $G$. [32] The Cartesian product $H_{q_{1}, \ldots, q_{d}}=K_{q_{1}} \square \cdots \square K_{q_{d}}$ of complete graphs is called a Hamming graph where $q_{1}, \ldots, q_{d} \geq 2$ are integers. When $q_{1}=\cdots=q_{d}=q$, we write $H(d, q)$ in place of $H_{d}^{q, \ldots, q}$.

Theorem 4.45. [19] Suppose $d, q \geq 2$ are integers such that $q$ is a divisor of $d$. Then $H(d, q)$ is $\mathbb{Z}_{q}^{d}$-distance magic with magic constant $\left(\frac{q}{2}, \ldots, \frac{q}{2}\right)$ when $q$ is even and $(0, \ldots, 0)$ when $q$ is odd.

Lemma 4.46. [14] If $m, n$ are odd, then $C_{m} \square C_{n}$ is not a $\Gamma$-distance magic graph for any Abelian group $\Gamma$ of order $m$.

Theorem 4.47. [14] Let $k=\operatorname{lcm}(m, n)$. If $m$ or $n$ is even, then $C_{m} \square C_{n}$ has a $\mathbb{Z}_{\alpha} \times \mathcal{A}$-distance magic labeling for any $\alpha \equiv 0(\bmod k)$ and any Abelian group $\mathcal{A}$ of order $\frac{m n}{\alpha}$.

Theorem 4.48. [19] Let $G_{i}$ be an $r_{i}$-regular graph with $n_{i} \geq 2$ vertices, $1 \leq i \leq k$. Let $\Gamma_{i}$ be an Abelian group of order $n_{i}$ such that $\exp \left(\Gamma_{i}\right)$ is a divisor of $r-r_{i}, 1 \leq i \leq k$, where $r=\sum_{i=1}^{k} r_{i}$. If $G_{i}$ is $\Gamma_{i}$-distance magic for $1 \leq i \leq k$, then $G_{1} \square \cdots \square G_{k}$ is $\Gamma_{1} \times \cdots \times \Gamma_{k}$-distance magic.

Corollary 4.49. [19] Let $d_{i}, r_{i} \geq 1$ and $n_{i} \geq 2$ be integers, $1 \leq i \leq k$. Let $G_{i}$ be an $r_{i}$-regular graph with $n_{i}^{d_{i}}$ vertices, $1 \leq i \leq k$. Suppose $n_{i}$ is a divisor of $r-r_{i}$, $1 \leq i \leq k$, where $r=\sum_{i=1}^{k} r_{i}$. If $G_{i}$ is $\mathbb{Z}_{n_{i}}^{\bar{d}_{i}}$-distance magic for $1 \leq i \leq k$, then $G_{1} \square \cdots \square G_{k}$ is $\mathbb{Z}_{n_{1}}^{d_{1}} \times \cdots \times \mathbb{Z}_{n_{k}}^{d_{k}}$-distance magic.

Corollary 4.50. [19] Let $G, H$ be regular graphs with $2^{d}, 2^{e}$ vertices respectively. Suppose both $G$ and $H$ have even degrees. If $G$ is $\mathbb{Z}_{2}^{d}$-distance magic and $H$ is $\mathbb{Z}_{2}^{e}$ distance magic, then $G \square H$ is $\mathbb{Z}_{2}^{d+e}$-distance magic.

Corollary 4.51. [19] Let $p_{i} \geq 2$ be a prime and $d_{i}, r_{i} \geq 1$ be integers with $2 \leq r_{i} \leq p_{i}^{d_{i}}$ for $i=1,2$. Let $G_{i}$ be any Cayley graph on $\mathbb{Z}_{p_{i}}^{d_{i}}$, with degree $r_{i}$ for $i=1,2$. If both $p_{1}$ and $p_{2}$ divide each of $r_{1}$ and $r_{2}$, then $G_{1} \square G_{2}$ is $\mathbb{Z}_{p_{1}}^{d_{1}} \times \mathbb{Z}_{p_{2}}^{d_{2}}$-distance magic.

An involution is a group element of order 2.

Theorem 4.52. [17] Let $G$ be an r-regular distance magic graph on $n$ vertices, where $r$ is odd. There does not exist an Abelian group $\Gamma$ of order $n$ having exactly one involution $\iota$ such that $G$ is $\Gamma$-distance magic. 
Observation 4.53. [17] Let $G$ be an $r$-regular distance magic graph on $n \equiv 2$ (mod 4) vertices, where $r$ is odd. There does not exist an Abelian group $\Gamma$ of order $n$ such that $G$ is $\Gamma$-distance magic.

Observation 4.54. [17] If $C_{n}(1, d)$ is a $\Gamma$-distance magic circulant graph for a group $\Gamma$, then $n$ is even.

Theorem 4.55. [17] If $\operatorname{gcd}(n, d+1)=2 k+1, d$ and $n$ are both even, and $n=$ $2 s(2 k+1)$ then $C_{n}(1, d)$ has a $\mathbb{Z}_{2 m} \times \mathcal{A}$-magic labeling for any $m \equiv 0(\bmod s)$ and any Abelian group $\mathcal{A}$ of order $\frac{s(2 k+1)}{m}$.

Corollary 4.56. [17] If $d$ is odd and $2 d^{2}-2 \not \equiv 0(\bmod n)$, then $C_{n}(1, d)$ is not $\Gamma$-distance magic for any Abelian group $\Gamma$ of order $n$.

Observation 4.57. [17] If $d=5$, then $C_{d^{2}-1}(1, d)$ is $\Gamma$-distance magic for any Abelian group $\Gamma$ of order $d^{2}-1$.

Observation 4.58. [14] If $n<2 d+2$, then $C_{n}^{d}$ is not a $\Gamma$-distance magic graph for any Abelian group $\Gamma$ of order $n$.

Observation 4.59. [14] If $C_{n}^{d}$ is a $\Gamma$-distance magic graph for a group $\Gamma$ then $n$ is even.

Theorem 4.60. [14] Let $\operatorname{gcd}(n, d+1)=k$. If $d$ is even, $n>2 d+1$, and $n=2 h k$, then $C_{n}^{d}$ has a $\mathbb{Z}_{\alpha} \times \mathcal{A}$-distance magic labeling for any $\alpha \equiv 0(\bmod 2 h)$ and any Abelian group $\mathcal{A}$ of order $\frac{n}{\alpha}$.

Lemma 4.61. [14] If $d$ is odd and $2 d(d+1) \not \equiv 0(\bmod n)$, then $C_{n}^{d}$ is not a $\Gamma$-distance magic graph for any Abelian group $\Gamma$ of order $n$.

Theorem 4.62. [14] Let $\operatorname{gcd}(n, d+1)=k$. If $d$ is odd, $n=2 h k, p \equiv 0(\bmod k)$, and $n>2 d+1$, then $C_{n}^{d}$ has a $\mathbb{Z}_{\alpha} \times \mathcal{A}$-distance magic labeling for any $\alpha \equiv 0(\bmod 2 h)$ and any Abelian group $\mathcal{A}$ of order $\frac{n}{\alpha}$.

\subsection{Group Distance Antimagic Graphs}

A $\Gamma$-distance antimagic labeling applies to graphs labelled with elements from Abelian group $\Gamma$, much like $\Gamma$-distance magic labelings or group distance magic labelings. [19]

\subsection{1 $\mathbb{Z}_{n}$-Distance Antimagic Graphs}

Corollary 4.63. [19] Let $G$ be an r-regular graph on $n$ vertices such that both $n$ and $r$ are even. Then $G$ is not $\mathbb{Z}_{n}$-distance antimagic.

Theorem 4.64. [19] The circulant graph $\operatorname{Cay}\left(\mathbb{Z}_{n} ; S\right)$ is $\mathbb{Z}_{n}$-distance antimagic if one of the following holds: 
- $n$ is even, $n / 2 \in S, S$ contains $2 s$ even integers and $2 t$ odd integers other than $n / 2$, and $2(s-t)+1$ and $2(t-s)+1$ are both coprime to $n$;

- $|S|$ and $n$ are coprime.

On the other hand, if $|S|$ and $n$ are both even, then $C a y\left(\mathbb{Z}_{n} ; S\right)$ is not $\mathbb{Z}_{n}$-distance antimagic.

\subsection{2 $\quad \Gamma$-Distance Antimagic Graphs}

Theorem 4.65. [19] Let $G$ be an r-regular graph on $n$ vertices, where $n$ is even. Then for any Abelian group $\Gamma$ of order $n$ with exactly one involution, $G$ cannot be $\Gamma$-distance antimagic unless $r$ is odd.

Corollary 4.66. [19] Let $G$ be an r-regular graph on $n$ vertices such that $n \equiv 2$ (mod 4) and $r$ is even. There does not exist an Abelian group $\Gamma$ of order $n$ such that $G$ is $\Gamma$-distance antimagic.

Theorem 4.67. [19] Let $\Gamma$ be a finite Abelian group of order $n=|\Gamma|$, and $G=$ $C a y(\Gamma ; S)$, a Cayley graph on $\Gamma$ of degree $r=|S|$. If $n$ and $r$ are coprime, then $G$ is $\Gamma$-distance antimagic, and any automorphism of $\Gamma$ is an $\Gamma$-distance antimagic labeling of $G$.

Corollary 4.68. [19] Let $p \geq 2$ be a prime, and let $r \geq 2$ and $d \geq 1$ be integers. If $p$ is not a divisor of $r$, then any Cayley graph Cay $\left(\mathbb{Z}_{p}^{d} ; S\right)$ on $\mathbb{Z}_{p}^{d}$ with degree $r$ is $\mathbb{Z}_{p}^{d}$-distance antimagic and any automorphism $f$ of $\mathbb{Z}_{p}^{d}$ is a $\mathbb{Z}_{p}^{d}$-distance antimagic labeling.

Corollary 4.69. [19] Let $d \geq 2$ be an integer. Any Cayley graph $\operatorname{Cay}\left(\mathbb{Z}_{2}^{d} ; S\right)$ on $\mathbb{Z}_{2}^{d}$ with an odd degree is $\mathbb{Z}_{2}^{d}$-distance antimagic with any automorphism $f$ of $\mathbb{Z}_{2}^{d}$ as a $\mathbb{Z}_{2}^{d}$-distance antimagic labeling.

Corollary 4.70. [19] The d-dimensional hypercube $Q_{d}$ is $\mathbb{Z}_{2^{d}}$-distance antimagic if and only if $d$ is odd.

Corollary 4.71. [19] Let $d \geq 2$ be an integer. If $d$ is odd, then $Q_{d}$ is $\mathbb{Z}_{2}^{d}$-distance antimagic.

Theorem 4.72. [19] Let $d \geq 3$ be an integer. If $d$ is odd or $d \equiv 0(\bmod 4)$, then $Q_{d}$ is $\mathbb{Z}_{2}^{d}$-distance antimagic.

Remark 4.73. [19] If $Q_{k}$ is $\mathbb{Z}_{2}^{k}$-distance antimagic for some integer $k \geq 6$ with $k \equiv 2$ $(\bmod 4)$, then $Q_{d}$ is $\mathbb{Z}_{2}^{d}$-distance antimagic for every integer $d \geq k$ with $d \equiv 2(\bmod 4)$. In particular, if $Q_{6}$ is $\mathbb{Z}_{2}^{6}$-distance antimagic, then $Q_{d}$ is $\mathbb{Z}_{2}^{d}$-distance antimagic for all integers $d \geq 3$. The problem of determining if $Q_{6}$ is $\mathbb{Z}_{2}^{6}$-distance antimagic is restated in Open Problem 6.29. 
Theorem 4.74. [19] Let $d \geq 1$ and $q \geq 2$ be integers.

- If $d$ and $q$ are coprime, then the Hamming graph $H(d, q)$ is $\mathbb{Z}_{q^{d}}$-distance antimagic. In particular, $K_{q}$ is $\mathbb{Z}_{q}$-distance antimagic for any $q \geq 2$.

- If both $d$ and $q$ are even, then $H(d, q)$ is not $\mathbb{Z}_{q^{d}}$-distance antimagic.

Most of the work with group distance antimagic graphs involves graph products. Like in chapter 2 and earlier in this chapter, we separate these results by the specific graph product.

\section{Direct Product}

The direct product $G_{1} \times \cdots \times G_{d}$ of $d$ graphs $G_{1}, \ldots, G_{d}$ is defined to have vertex set $V\left(G_{1}\right) \times \cdots \times V\left(G_{d}\right)$ such that two vertices $\left(x_{1}, \ldots, x_{d}\right)$ and $\left(y_{1}, \ldots, y_{d}\right)$ are adjacent if and only if $x_{i}$ is adjacent to $y_{i}$ in $G_{i}$ for $i=1, \ldots, d$.

Theorem 4.75. [19] Let $G_{i}$ be an $r_{i}$-regular graph with $n_{i}$ vertices and $\Gamma_{i}$ an Abelian group of order $n_{i}, i=1, \ldots, d$. Denote $r=r_{1} \cdots r_{d}$. If $G_{i}$ is $\Gamma_{i}$-distance antimagic and $n_{i}$ and $r / r_{i}$ are coprime for $i=1, \ldots, d$, then $G_{1} \times \cdots \times G_{d}$ is $\Gamma_{1} \times \cdots \times \Gamma_{d}$-distance antimagic.

Let $G_{\times}^{d}=\underbrace{G \times \cdots \times G}_{d \text { times }}$ and $\Gamma_{\times}^{d}=\underbrace{\Gamma \times \cdots \times \Gamma}_{d \text { times }}$.

Corollary 4.76. [19] Suppose $G$ is an $r$-regular $\Gamma$-distance antimagic graph with $n$ vertices, where $\Gamma$ is an Abelian group of order $n$. If $n$ and $r$ are coprime, then for any integer $d \geq 1, G_{\times}^{d}$ is $\Gamma^{d}$-distance antimagic.

Corollary 4.77. [19] For any odd integers $n_{1}, \ldots, n_{d} \geq 3, C_{n_{1}} \times \cdots \times C_{n_{d}}$ is $\mathbb{Z}_{n_{1}} \times$ $\cdots \times \mathbb{Z}_{n_{d}}$-distance antimagic. In particular, for any integer $d \geq 1$ and odd integer $n \geq 3,\left(C_{n}\right)_{\times}^{d}$ is $\mathbb{Z}_{n}^{d}$-distance antimagic.

Corollary 4.78. [19] Let $n_{1}, \ldots, n_{d} \geq 3$, be integers such that $n_{i}$ and $n_{j}-1$ are coprime for distinct $i, j$. Then $K_{n_{1}} \times \cdots \times K_{n_{d}}$ is $\mathbb{Z}_{n_{1}} \times \cdots \times \mathbb{Z}_{n_{d}}$-distance antimagic. In particular, for any integers $d \geq 1$ and $n \geq 3,\left(K_{n}\right)_{\times}^{d}$ is $\mathbb{Z}_{n}^{d}$-distance antimagic.

Let $D_{n}=C_{n} \square P_{2}$, the prism of $2 n \geq 6$ vertices.

Lemma 4.79. [19] Let $n \geq 4$ be an integer not divisible by 3 . Then $D_{n}$ is $\mathbb{Z}_{2 n^{-}}$ distance antimagic.

Corollary 4.80. [19] Let $n_{1}, \ldots, n_{d} \geq 4$ be integers not divisible by 3 . Then $D_{n_{1}} \times$ $\cdots \times D_{n_{d}}$ is $\mathbb{Z}_{n_{1}} \times \cdots \times \mathbb{Z}_{n_{d}}$-distance antimagic. In particular, for any $d \geq 1$, and any $n \geq 4$ not divisible by $3,\left(D_{n}\right)_{\times}^{d}$ is $\mathbb{Z}_{n}^{d}$-distance antimagic. 
Theorem 4.81. [19] Let $G_{i}$ be an $r_{i}$-regular graph with $n_{i}$ vertices, $i=1, \ldots, k$. Suppose $n_{i}$ and $r / r_{i}$ are coprime for $i=1, \ldots, k$, where $r=r_{1} \cdots r_{k}$. If each $G_{i}$ is $\mathbb{Z}_{n_{i}}$-distance antimagic, then $G_{1} \times \cdots \times G_{k}$ is $\mathbb{Z}_{n_{1} \ldots n_{k}}$-distance antimagic.

Corollary 4.82. [19] Let $n_{1}, \ldots, n_{k} \geq 3$ be (not necessarily distinct) integers. Then $C_{n_{1}} \times \cdots \times C_{n_{k}}$ is $\mathbb{Z}_{n_{1} \ldots n_{k}}$-distance antimagic if and only if all $n_{1}, \ldots, n_{k}$ are odd. In particular, for any integer $d \geq 1$ and odd integer $n \geq 3,\left(C_{n}\right)_{\times}^{d}$ is $\mathbb{Z}_{n^{d}}$-distance antimagic.

\section{Cartesian Product}

Theorem 4.83. [19] Let $G_{i}$ be an $r_{i}$-regular graph with $n_{i} \geq 2$ vertices, $1 \leq i \leq k$. Let $\Gamma_{i}$ be an Abelian group of order $n_{i}$ such that $\exp \left(\Gamma_{i}\right)$ is a divisor of $r-r_{i}, 1 \leq i \leq k$, where $r=\sum_{i=1}^{k} r_{i}$. If $G_{i}$ is $\Gamma_{i}$-distance antimagic for $1 \leq i \leq k$, then $G_{1} \square \cdots \square G_{k}$ is $\Gamma_{1} \times \cdots \times \Gamma_{k}$-distance antimagic.

Corollary 4.84. [19] Let $d_{i}, r_{i} \geq 1$ and $n_{i} \geq 2$ be integers, $1 \leq i \leq k$. Let $G_{i}$ be an $r_{i}$-regular graph with $n_{i}^{d_{i}}$ vertices, $1 \leq i \leq k$. Suppose $n_{i}$ is a divisor of $r-r_{i}$, $1 \leq i \leq k$, where $r=\sum_{i=1}^{k} r_{i}$. If $G_{i}$ is $\mathbb{Z}_{n_{i}}^{d_{i}}$-distance antimagic for $1 \leq i \leq k$, then $G_{1} \square \cdots \square G_{k}$ is $\mathbb{Z}_{n_{1}}^{d_{1}} \times \cdots \times \mathbb{Z}_{n_{k}}^{d_{k}}$-distance antimagic.

Corollary 4.85. [19] Let $G, H$ be regular graphs with $2^{d}, 2^{e}$ vertices respectively. Suppose both $G$ and $H$ have even degrees. If $G$ is $\mathbb{Z}_{2}^{d}$-distance antimagic and $H$ is $\mathbb{Z}_{2}^{e}$-distance antimagic, then $G \square H$ is $\mathbb{Z}_{2}^{d+e}$-distance antimagic.

Corollary 4.86. [19] Let $p_{i} \geq 2$ be a prime and $d_{i}, r_{i} \geq 1$ be integers with $2 \leq r_{i} \leq p_{i}^{d_{i}}$ for $i=1,2$. Let $G_{i}$ be any Cayley graph on $\mathbb{Z}_{p_{i}}^{d_{i}}$ with degree $r_{i}$ for $i=1,2$. If $p_{1}$ divides $r_{2}$ but not $r_{1}$, and $p_{2}$ divides $r_{1}$ but not $r_{2}$, then $G_{1} \square G_{2}$ is $\mathbb{Z}_{p_{1}}^{d_{1}} \times \mathbb{Z}_{p_{2}}^{d_{2}}$-distance antimagic.

Corollary 4.87. [19] Let $d_{i} \geq 1$ and $q_{i} \geq 2$ be integers which are coprime, $1 \leq i \leq k$. If $q_{i}^{d_{i}}$ is a divisor of $\sum_{j \neq i} d_{j}\left(q_{j}-1\right)$ for $1 \leq i \leq k$, then $H\left(d_{1}, q_{1}\right) \square \cdots \square H\left(d_{k}, q_{k}\right)$ is $\mathbb{Z}_{q_{1}^{d_{1}}} \times \cdots \times \mathbb{Z}_{q_{k}^{d_{k}}}$-distance antimagic.

Theorem 4.88. [19]

- If $n \geq 3$ is an odd integer, then $C_{n}$ is $\mathbb{Z}_{n}$-distance antimagic.

- Let $n_{1}, \ldots n_{k} \geq 3$ be odd integers, where $k \geq 2$. If each $n_{i}$ is a divisor of $k-1$, then $C_{n_{1}} \square \cdots \square C_{n_{k}}$ is $\mathbb{Z}_{n_{1}} \times \cdots \times \mathbb{Z}_{n_{k}}$-distance antimagic. In particular, for any prime $p$ and any integer $d \geq 1, C_{p} \square \cdots \square C_{p}$ (pd +1 factors) is $\mathbb{Z}_{p}^{p d+1}$-distance antimagic.

Given an $r$-regular graph $G$ with $n$ vertices and an Abelian group $\Gamma$ of order $n$, a bijection $f: V(G) \rightarrow \Gamma$ is called an $\Gamma$-balanced labeling if $w(x)=r f(x)$ for every $x \in V(G)$. 
Theorem 4.89. [19] Let $G_{i}$ be an $r_{i}$-regular graph with $n_{i} \geq 2$ vertices, $1 \leq i \leq k$. Let $\Gamma_{i}$ be an Abelian group of order $n_{i}, 1 \leq i \leq k$, and let $r=\sum_{i=1}^{k} r_{i}$. Suppose $\{1, \ldots, k\}$ is partitioned into, say, $I=\left\{1, \ldots, k_{1}\right\}, J=\left\{k_{1}+1, \ldots, k_{2}\right\}$, and $L=$ $\left\{k_{2}+1, \ldots, k\right\}$ for some $0 \leq k_{1} \leq k_{2} \leq k$, possibly with one or two of $I, J, L$ to be empty, such that the following conditions are satisfied:

- for each $h \in H, G_{h}$ is $\Gamma_{h}$-distance magic and $r-r_{h}$ is coprime to $n_{h}$;

- for each $i \in I, G_{i}$ is $\Gamma_{i}$-distance antimagic and $\exp \left(\Gamma_{i}\right)$ is a divisor of $r-r_{i}$;

- for each $j \in J, G_{j}$ admits an $\Gamma_{j}$-balanced labeling and $r$ is coprime to $n_{j}$.

Then $G_{1} \square \cdots \square G_{k}$ is $\Gamma_{1} \times \cdots \times \Gamma_{k}$-distance antimagic.

Corollary 4.90. [19] Suppose $G_{i}$ is an $r_{i}$-regular $\mathbb{Z}_{2}^{d_{i}}$-distance magic graph, $1 \leq$ $i \leq s$, and $G_{j}$ an $r_{j}$-regular $\mathbb{Z}_{2}^{d_{j}}$-distance antimagic graph, $s+1 \leq j \leq s+t$. Let $r=\sum_{i=1}^{s+t} r_{i}$ and $d=\sum_{i=1}^{s+t} d_{i}$. If $r_{i}$ and $r$ have different parity for $i=1, \ldots, s$ and the same parity for $i=s+1, \ldots, s+t$, then $G_{1} \square \cdots \square G_{s+t}$ is $Z_{2}^{d}$-distance antimagic. In particular, if $G_{1}$ is a regular $\mathbb{Z}_{2}^{d_{1}}$-distance magic graph with even degree, and $G_{2}$ is a regular $\mathbb{Z}_{2}^{d_{2}}$-distance antimagic graph with odd degree, then $G_{1} \square G_{2}$ is $\mathbb{Z}_{2}^{d_{1}+d_{2}}$-distance antimagic.

Theorem 4.91. [19] Suppose $G_{i}$ is an $r_{i}$-regular graph with $n_{i}$ vertices which admits $a \mathbb{Z}_{n}$-balanced labeling, $1 \leq i \leq k$. Suppose further that $r_{i} \leq n_{j}$ for any $1 \leq i, j, \leq k$ and that $r=\sum_{i=1}^{k} r_{i}$ is coprime to $n_{1} \ldots n_{k}$. Then $G_{1} \square \cdots \square G_{k}$ is $\mathbb{Z}_{n_{1} \ldots n_{k}}$-distance antimagic.

Denote $G_{\square}^{k}=\underbrace{G \square \cdots \square G}_{k \text { times }}$.

Corollary 4.92. [19] Let $n_{1}, \ldots, n_{k} \geq 3$ be (not necessarily distinct) integers such that $k$ is coprime to $n_{1} \cdots n_{k}$. Then $C_{n_{1}} \square \cdots \square C_{n_{k}}$ is $\mathbb{Z}_{n_{1} \ldots n_{k}}$-distance antimagic if and only if all $n_{1}, \ldots, n_{k}$ are odd. In particular, for any integer $k \geq 1$ and odd integer $n \geq 3$ that are coprime, $\left(C_{n}\right)_{\square}^{k}$ is $\mathbb{Z}_{n^{k}}$-distance antimagic.

Corollary 4.93. [19] Let $p$ be a prime and let $n_{1}, \ldots, n_{p} \geq 3$ be (not necessarily distinct) integers none of which has $p$ as a factor. Then $C_{n_{1}} \square \cdots \square C_{n_{p}}$ is $\mathbb{Z}_{n_{1} \ldots n_{p}}$ distance antimagic if and only if all $n_{1}, \ldots, n_{p}$ are odd. In particular, for any integers $n_{1}, n_{2} \geq 3, C_{n_{1}} \square C_{n_{2}}$ is $\mathbb{Z}_{n_{1} n_{2}}$-distance antimagic if and only if both $n_{1}$ and $n_{2}$ are odd. Moreover, if a prime $p$ is not a divisor of an odd integer $n \geq 3$, then $\left(C_{n}\right)_{\square}^{p}$ is $\mathbb{Z}_{n^{p}}$-distance antimagic.

\section{Strong Product}

The strong product, denoted $G \otimes H$ is a graph with vertex set $V(G) \times V(H)$. Vertices $(g, h)$ and $\left(g^{\prime}, h^{\prime}\right)$ in $G \otimes H$ are adjacent if and only if $g=g^{\prime}$ and $h$ is adjacent to $h^{\prime}$ in $H$, or $h=h^{\prime}$ and $g$ is adjacent to $g^{\prime}$ in $G$, or $g$ is adjacent to $g^{\prime}$ in $G$ and $h$ is adjacent to $h^{\prime}$ in $H$. [32] 
Theorem 4.94. [19] Let $G_{i}$ be an $r_{i}$-regular graph with $n_{i}$ vertices for $i=1,2$.

- Suppose both $G_{1}$ and $G_{2}$ have $\mathbb{Z}_{n}$-balanced labelings and $r_{1} r_{2}+r_{1}+r_{2}$ is coprime to $n_{1} n_{2}$. Then $G_{1} \otimes G_{2}$ is $\mathbb{Z}_{n_{1} n_{2}}$-distance antimagic.

- Suppose $G_{1}$ is $\mathbb{Z}_{n_{1}}$-distance magic, $G_{2}$ is $\mathbb{Z}_{n_{2}}$-distance magic, $r_{1}$ is coprime to $n_{2}$, and $r_{2}$ is coprime to $n_{1}$. Then $G_{1} \otimes G_{2}$ is $\mathbb{Z}_{n_{1} n_{2}}$-distance antimagic.

Corollary 4.95. [19] Let $m, n \geq 3$ be (not necessarily distinct) integers. Then $C_{m} \otimes C_{n}$ is $\mathbb{Z}_{m n}$-distance antimagic if and only if both $m$ and $n$ are odd.

Corollary 4.96. [19] For any odd integers $m, n \geq 3$ (not necessarily distinct), $K_{m} \otimes K_{n}$ is $\mathbb{Z}_{m n}$-distance antimagic.

Theorem 4.97. [19] Let $G$ be a regular graph on $m$ vertices other than the empty $\operatorname{graph} \bar{K}_{m}$.

- If $n$ is even, then $K_{n} \otimes G$ is $\mathbb{Z}_{n m}$-distance antimagic.

- If both $n$ and $m$ are odd, then $K_{n} \otimes G$ is $\mathbb{Z}_{n m}$-distance antimagic. 


\section{Chapter 5}

\section{Applications}

One important way that distance magic and distance antimagic labelings can be used is in the scheduling of tournaments. A fair way to schedule a tournament is to create a round-robin, where each team plays every other team once. However, playing that many games is not always possible. Thus, we need a fair way of scheduling when not all of the games from the round-robin can be played. In order to be fair, each team should play the same number of teams and the difficulty of the schedule for each team should mimic the difficulty of playing the entire round-robin tournament. To assist with considering the difficulty of each team's schedule, if there are $n$ teams in the tournament, rank the teams from strongest to weakest so that the strongest team has rank 1 and the weakest team has rank $n$. We define the strength of the $i$-th ranked team as $s_{n}(i)=n+1-i$. Thus the strongest team will have rank 1 and strength $n$. Additionally, the total strength of opponents of team $i$ in the complete round-robin tournament is defined as $S_{n, n-1}(i)=\frac{n(n+1)}{2}-s_{n}(i)=\frac{(n+1)(n-2)}{2}+i$.

One way to create a fair schedule is to create a fair incomplete tournament. In a fair incomplete tournament of $n$ teams with $k$ rounds, written $\operatorname{FIT}(n, k)$, every team plays exactly $k$ other teams, and the total strength of the opponents team $i$ plays is

$S_{n, k}(i)=\frac{(n+1)(n-2)}{2}+i-m$ where $m$ is a fixed constant. In such a tournament, the schedule strength each team misses is the same, namely $m$.

Another way to create a fair schedule is to create an equalized incomplete tournament. An equalized incomplete tournament of $n$ teams with $r$ rounds, written $\operatorname{EIT}(n, r)$ is a tournament in which every team plays exactly $r$ other teams and the total strength of the opponents team $i$ plays is $T_{n, r}(i)=m$ for every $i$, where $m$ is a fixed constant. In such a tournament, each team has the same strength of schedule.

Certainly, we can see that $\operatorname{EIT}(n, n-k-1)$ is the complement of $F \operatorname{IT}(n, k)$ and thus $\operatorname{FIT}(n, k)$ exists if and only if $\operatorname{EIT}(n, n-k-1)$ exists.

Lemma 5.1. [27] Let EIT $(n, r)$ be an equalized tournament. Then $r$ is even.

Theorem 5.2. [27] Let EIT $(n, r)$ be an equalized tournament with an even number of teams, $n$, and $r$ rounds, where $r \equiv 2(\bmod 4)$. Then $n \equiv 0(\bmod 4)$. 
Table 5.1: Equalized Incomplete Tournament of 6 Rounds for 12 teams

\begin{tabular}{|l|l|l|l|l|l|l|}
\hline Team & Round $\mathbf{1}$ & Round 2 & Round 3 & Round 4 & Round 5 & Round 6 \\
\hline 1 & 2 & 4 & 6 & 7 & 9 & 11 \\
\hline 2 & 1 & 3 & 5 & 8 & 10 & 12 \\
\hline 3 & 4 & 2 & 7 & 9 & 11 & 6 \\
\hline 4 & 3 & 1 & 8 & 10 & 12 & 5 \\
\hline 5 & 9 & 6 & 2 & 11 & 7 & 4 \\
\hline 6 & 10 & 5 & 1 & 12 & 8 & 3 \\
\hline 7 & 12 & 10 & 3 & 1 & 5 & 8 \\
\hline 8 & 11 & 9 & 4 & 2 & 6 & 7 \\
\hline 9 & 5 & 8 & 12 & 3 & 1 & 10 \\
\hline 10 & 6 & 7 & 11 & 4 & 2 & 9 \\
\hline 11 & 8 & 12 & 10 & 5 & 3 & 1 \\
\hline 12 & 7 & 11 & 9 & 6 & 4 & 2 \\
\hline
\end{tabular}

Theorem 5.3. [27] For $n$ even, an $\operatorname{EIT}(n, r)$ exists if and only if $2 \leq r \leq n-2$, $r \equiv 0(\bmod 2)$ and either $n \equiv 0(\bmod 4)$ or $n \equiv r+2 \equiv 2(\bmod 4)$.

Corollary 5.4. [27] For $n$ even, a FIT $(n, r)$ exists if and only if $1 \leq r \leq n-1$, $k \equiv 1(\bmod 2)$ and either $n \equiv 0(\bmod 4)$ or $n \equiv k+1 \equiv 2(\bmod 4)$.

For example, suppose you want to schedule a tournament with 12 teams where each team plays 6 games. An $\operatorname{EIT}(12,6)$ providing the opponent for each team in each round is displayed in Table 5.1. The fixed constant $m$ in this case is 39. Finding an $\operatorname{EIT}(12,6)$ is equivalent to finding a distance magic labeling for the 6-regular graph on 12 vertices with magic constant 39.

Similarly, finding a FIT $(12,5)$ is equivalent to taking the complement of a 6 regular graph on 12 vertices. Table 5.2 provides the opponent for each team in each round.

Now $\operatorname{EIT}(n, r)$ and $F I T(n, k)$ both attempt to replicate the results of performing the full round-robin tournament. Of course, a strong team is likely to win more games than a weak team in such a tournament. If we want all teams to have an equal chance of winning the tournament, we can create a handicap incomplete tournament. A handicap incomplete tournament of $n$ teams with $r$ rounds, $H I T(n, r)$, is a tournament where each team $i$ plays $r$ opponents with a combined strength of $S_{n, r}(i)=t-i$, where $t$ is a fixed constant. (This means the strongest team will play the strongest opponents and the weakest team will play the weakest opponents.)

A special case is a handicap distance $d$-antimagic labeling of a graph $G(V, E)$ with $n$ vertices, which is a bijection $\vec{f}: V \rightarrow\{1,2, \ldots, n\}$ with the property that $\vec{f}\left(x_{i}\right)=i$ and the sequence of the weights $w\left(x_{1}\right), w\left(x_{2}\right), \ldots w\left(x_{n}\right)$ forms an increasing arithmetic progression with difference $d$. A graph $G$ is a handicap distance $d$-antimagic graph if 
Table 5.2: Fair Incomplete Tournament of 5 Rounds for 12 teams

\begin{tabular}{|l|l|l|l|l|l|}
\hline Team & Round $\mathbf{1}$ & Round 2 & Round $\mathbf{3}$ & Round 4 & Round 5 \\
\hline 1 & 12 & 3 & 10 & 5 & 8 \\
\hline 2 & 11 & 4 & 9 & 6 & 7 \\
\hline 3 & 5 & 1 & 12 & 8 & 10 \\
\hline 4 & 7 & 2 & 11 & 9 & 6 \\
\hline 5 & 3 & 10 & 8 & 1 & 12 \\
\hline 6 & 9 & 11 & 7 & 2 & 4 \\
\hline 7 & 4 & 9 & 6 & 11 & 2 \\
\hline 8 & 10 & 12 & 5 & 3 & 1 \\
\hline 9 & 6 & 7 & 2 & 4 & 11 \\
\hline 10 & 8 & 5 & 1 & 12 & 3 \\
\hline 11 & 2 & 6 & 4 & 7 & 9 \\
\hline 12 & 1 & 8 & 3 & 10 & 5 \\
\hline
\end{tabular}

it allows a handicap distance $d$-antimagic labeling and handicap distance antimagic graph when $d=1$. [23]

Theorem 5.5. [23] Let $c\left(K_{a} \square K_{b}\right)$ denote the graph of c mutually disjoint copies of the Cartesian product of complete graphs $K_{a}$ and $K_{b}$. Let $a$ and $b$ be even positive integers such that $2 \leq a \leq b, 4<a b$, and $c$ be any positive integer. Let $n=a b c$ and $G=c\left(K_{a} \square K_{b}\right)$. Then the complement of $G$ is a handicap distance antimagic graph with $n$ vertices.

Theorem 5.6. [25] Let $a, b, c$ be positive odd integers such that $1<a \leq b$, and $q>1$ divides at least two of $a, b, c$. let $n=a b c$ and $G=c\left(K_{a} \square K_{b}\right)$. Then the complement of $G$ is a handicap distance antimagic graph with $n$ vertices.

For example, suppose you want to schedule a tournament with 12 teams where each team plays 5 games. A $\operatorname{HIT}(12,5)$ providing the opponent for each team in each round is displayed in Table 5.3. The fixed constant $t=39$.

Let $Q_{n}$ denote the hypercube with the vertex set of all binary $n$-tuples where vertices are adjacent if and only if they differ in exactly one position.

Theorem 5.7. [25] The graph $G=c Q_{3}$ is handicap distance antimagic for any $c \geq 1$.

Theorem 5.8. [25] Let $Q_{4}^{M}$ be the graph arising from $Q_{4}$ by adding the perfect matching consisting of edges $\left(x_{0} x_{1} x_{2} x_{3}, y_{0} y_{1} y_{2} y_{3}\right)$ satisfying $x_{i}+y_{i} \equiv 1(\bmod 2)$ for $i=0,1,2,3$. Then $c Q_{4}^{M}$ is handicap distance antimagic for any $c \geq 1$.

Observation 5.9. [25] Let $G$ be a handicap distance antimagic graph and $H$ a distance magic graph such that $V(G)=V(H)$ and $E(G) \cap E(H)=\emptyset$. Then the 
Table 5.3: Handicap Incomplete Tournament of 5 Rounds for 12 teams

\begin{tabular}{|l|l|l|l|l|l|}
\hline Team & Round $\mathbf{1}$ & Round 2 & Round $\mathbf{3}$ & Round $\mathbf{4}$ & Round 5 \\
\hline 1 & 11 & 3 & 9 & 7 & 8 \\
\hline 2 & 12 & 10 & 4 & 5 & 6 \\
\hline 3 & 9 & 1 & 8 & 11 & 7 \\
\hline 4 & 10 & 5 & 2 & 6 & 12 \\
\hline 5 & 6 & 4 & 12 & 2 & 10 \\
\hline 6 & 5 & 12 & 10 & 4 & 2 \\
\hline 7 & 8 & 9 & 11 & 1 & 3 \\
\hline 8 & 7 & 11 & 3 & 9 & 1 \\
\hline 9 & 3 & 7 & 1 & 8 & 11 \\
\hline 10 & 4 & 2 & 6 & 12 & 5 \\
\hline 11 & 1 & 8 & 7 & 3 & 9 \\
\hline 12 & 2 & 6 & 5 & 10 & 4 \\
\hline
\end{tabular}

graph $G \boxplus H$ with $V(G \boxplus H)=V(G)=V(H)$ and $E(G \boxplus H)=E(G) \bigcup E(H)$ is handicap distance antimagic. 


\section{Chapter 6}

\section{Open Problems}

In this chapter, we list problems that, as far as we can determine, remain unsolved.

Seoud et. al [51] posed the following problem.

Problem 6.1. Classify whether or not the following are distance magic graphs:

- $K_{n} \square C_{m}$ where $n \geq 2, m \geq 3$;

- $C_{m} \square P_{n}$ where $n \geq 2, m \geq 3$; and

- $C_{m} \square K_{n, t}$, where $m \geq 3, n, t \geq 1$.

S.B. Rao et. al. ask in [48] to

Problem 6.2. Characterize 4-regular distance magic graphs. ${ }^{1}$

Kovar and Silber posed this problem on regular graphs in [38].

Problem 6.3. For what values of $n$ do $(n-5)$-regular distance graphs exist?

Kovar et. al. conjectured the following in [39]:

Conjecture 6.4. For all $n \geq 14$ an $r$-regular distance magic graph with $n$ vertices exists

- for all $14 \leq r \leq n-3$ if $n \equiv 0(\bmod 3)$

- for all $14 \leq r \leq n-5$ otherwise.

Cichacz [13] asks,

Problem 6.5. Decide whether a $(t-1, t)$-hypercycle of order $n$ is a distance magic hypergraph when $t>3$ is odd.

Gregor and Kovar [30] posed the next two questions:

\footnotetext{
${ }^{1}$ This problem is partially addressed by [17] and [37].
} 
Conjecture 6.6. For every neighbor-balanced $f: F_{2}^{n} \rightarrow F_{2}^{n}$ there is a regular balanced $n \times n$ matrix $M$ and $v \in F_{2}^{n}$ such that $f(u)=\left(M u^{T}\right) \oplus v$ for every $u \in F_{2}^{n}$.

Problem 6.7. Find a distance magic labeling of $Q_{n}$ for some $n \equiv 2(\bmod 4)$ that is not neighbor-balanced.

Acharya et. al. ask [2],

Conjecture 6.8. For any even integer $n \geq 4$, the $n$-dimensional hypercube $Q_{n}$ is not a distance magic graph.

This conjecture was posed by Miller, et. al. [40] and relates to complete multipartite graphs.

Conjecture 6.9. Let $1 \leq n_{1} \leq \cdots \leq n_{m}, m>1$. Let $s_{i}=\sum_{j=1}^{i} n_{j}$ and $n=s_{m}$. There exists a distance magic labeling of the complete multipartite graph $K_{n_{1}, \ldots, n_{m}}$ if and only if the following conditions hold:

- $a_{2} \geq 2$,

- $n(n+1) \equiv 0(\bmod 2 m)$, and

- $\sum_{j=1}^{s_{i}}(n+1-j) \geq \frac{i n(n+1)}{2 m}$ for $1 \leq i \leq m$.

By Theorem 2.49 and Theorem 2.56, we know this conjecture is valid for $m=$ $\{2,3\}$, but it is open for other values of $m$.

An independent set is a set of vertices, no two of which are adjacent. [11] Sugeng et. al. ask, [54]

Conjecture 6.10. If $G$ is a distance magic graph different from $K_{1,2,2, \ldots, 2}$, then the vertex set $V$ can be partitioned into sets $V_{1}, V_{2}, \ldots, V_{m}$ such that for each $i$, has $\left|V_{i}\right|>1$ and $V_{i}$ is independent.

Shafiq et. al. ask in [52]

Problem 6.11. If $G$ is a non-regular graph, determine if there is a distance magic labeling of $G \circ C_{4} .^{2}$

Anholcer et. al. ask in [5]

Problem 6.12. If $G$ is a non-regular graph, determine if there is a distance magic labeling of $G \times C_{4} \cdot{ }^{3}$

Rao asks [47],

\footnotetext{
${ }^{2}$ This problem is partially addressed by [12], [16], and [4].

${ }^{3}$ This is partially addressed by [12].
} 
Problem 6.13. Characterize graphs $G$ and $H$ such that $G \square H$ is a distance magic graph. $^{4}$

Arumugum et. al. [7] ask,

Problem 6.14. Does the adjacency matrix of a distance magic graph have special properties? ${ }^{5}$

Arumugam et. al. posed the following 2 problems in [8],

Problem 6.15. Does there exist a distance magic graph of order $n \equiv 1(\bmod 4)$ with magic constant $k=j n$, where $j$ is an odd integer with $3 \leq j \leq \frac{n-1}{2}$ ?

Problem 6.16. Determine the set

$$
S=\{k \in \mathbb{N} \text { : there exists a distance magic graph with magic constant } k\} .
$$

It is known that $1,2,4,6,8,12 \notin S$ and integers $4 n+3 \in S$ for all $n \geq 0$, integers $4 n+1 \in S$ for all $n \geq 1$, and $2^{n} \in S$ for every $n \geq 6$.

O'Neal and Slater [44] ask

Problem 6.17. Which weight sets $W$ allow a neighborhood spread of 0 ; that is, $N S_{W}^{s p}(G ; D)=0$ ?

O'Neal and Slater [44] ask

Problem 6.18. Why are multiple edge magic constants are possible for a graph when the vertex magic constant is unique?

Simanjuntak et. al. pose the following seven problems in [53]

Problem 6.19. Let $G$ be a non-connected graph having connected components $G_{1}, G_{2}, \ldots, G_{p}$, each of diameter $d_{1}, d_{2}, \ldots, d_{p}$ respectively. Let $d_{\max }=\max d_{i}$ and let there exist $i, j$ such that $\left|V\left(G_{i}\right)\right| \neq\left|V\left(G_{j}\right)\right|$. Does there exist $G$ admitting a $\left\{0,1, \ldots, d_{\max }\right\}$-distance magic labeling other than $K_{2} \cup K_{1}$ ?

Problem 6.20. Given a particular distance set D, what are the necessary and suffcient conditions for 2-regular graphs to have a D-distance magic labeling?

Problem 6.21. What are the necessary and sufficient conditions for trees to have $D$-distance magic labelings where $D \subseteq\{0,1,2\}$ ?

Conjecture 6.22. Graphs with diameter 2 are not $\{2\}$-distance magic. More generally, graphs with diameter $d$ are not $\{d\}$-distance magic.

\footnotetext{
${ }^{4}$ This is partially answered by Rao in [48].

${ }^{5}$ This is partially addressed by [41].
} 
Problem 6.23. Does there exist a graph of diameter larger than 2 other than $P_{4}$ admitting a $\{0,2\}$-distance magic labeling?

Problem 6.24. Does there exist an infinite family of non-regular graphs admitting a $\{1,2\}$-distance magic labeling?

Problem 6.25. Does there exist an infinite family of graphs with diameter at least 4 admitting a $\{1,2\}$-distance magic labeling?

In [22], Froncek asks the following:

Problem 6.26. For a given graph $C_{m} \square C_{n}$, determine all Abelian groups $\Gamma$ such that the graph $C_{m} \square C_{n}$ admits a $\Gamma$-distance magic labeling. ${ }^{6}$

In [19], Cichacz, et. al. ask the following five problems:

Problem 6.27. When does a Cayley graph on $\mathbb{Z}_{2}^{d}$ with even degree (odd degree, respectively) admit a $\mathbb{Z}_{2}^{d}$-distance antimagic (magic, respectively) labeling?

Problem 6.28. Give a necessary and sufficient condition for the Hamming graph

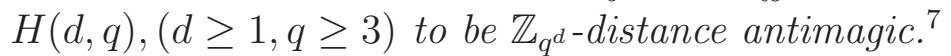

Problem 6.29. Prove or disprove $Q_{6}$ is $\mathbb{Z}_{2}^{6}$-distance antimagic.

Problem 6.30. Give a necessary and sufficient condition for $C_{n_{1}} \square \cdots \square C_{n_{d}}$ to be $\mathbb{Z}_{n_{1} \ldots n_{d}}$-distance antimagic when $d$ is not coprime to $n_{1} \cdots n_{d}$. (A necessary condition is that all $n_{i}$ 's must be odd.)

Problem 6.31. Give a necessary and sufficient condition for a circulant graph on $n$ vertices to be $\mathbb{Z}_{n}$-distance antimagic.

In [19], Cichacz, et. al. note

Problem 6.32. If $Q_{k}$ is $\mathbb{Z}_{2}^{k}$-distance antimagic for some integer $k \geq 6$ with $k \equiv$ $2(\bmod 4)$, then $Q_{d}$ is $\mathbb{Z}_{2}^{d}$-distance antimagic for every integer $d \geq k$ with $d \equiv 2$ $(\bmod 4)$; that is, if $Q_{6}$ is $\mathbb{Z}_{2}^{6}$-distance antimagic, then $Q_{d}$ is $\mathbb{Z}_{2}^{d}$-distance antimagic for all integers $d \geq 3$.

Anholcer et. al. conjecture [3],

Conjecture 6.33. If $m, n \equiv 0(\bmod 4)$, then $C_{m} \times C_{n}$ is a group distance magic graph.

Anholcer et. al. conjecture [3],

\footnotetext{
${ }^{6}$ This is partially addressed by [14].

${ }^{7}$ This is partially addressed by [19].
} 
Conjecture 6.34. Assume that $m, n \geq 3$ and $\{m, n\}=\{4 a, 4 b+c\}$ for some integers $a \geq 3$ and $b \geq 0, c \in\{1,2,3\}$. Then $C_{m} \times C_{n}$ is $\Gamma$-distance magic in the following cases:

- $c \in\{1,3\}$ and $\Gamma \cong \mathcal{A} \times \mathbb{Z}_{3} \times\left(\mathbb{Z}_{2}\right)^{p}$ for some Abelian group $\mathcal{A}$ of odd order, where $a=3 \cdot 2^{p-2}$,

- $c=2$ and $\Gamma \cong \mathcal{A} \times \mathbb{Z}_{3} \times\left(\mathbb{Z}_{2}\right)^{p+1}$ for some Abelian group $\mathcal{A}$ of odd order, where $a=3 \cdot 2^{p-2}$,

- $c=2$ and $\Gamma \cong \mathcal{A} \times \mathbb{Z}_{3} \times\left(\mathbb{Z}_{2}\right)^{p-1} \times \mathbb{Z}_{4}$ for some Abelian group $\mathcal{A}$ of odd order, where $a=3 \cdot 2^{p-2}$,

- $c=2$ and $\Gamma \cong \mathcal{A} \times \mathbb{Z}_{5} \times\left(\mathbb{Z}_{2}\right)^{p}$ for some Abelian group $\mathcal{A}$ of odd order, where $a=5 \cdot 2^{p-3}$,

- $c=2$ and $\Gamma \cong \mathcal{A} \times \mathbb{Z}_{7} \times\left(\mathbb{Z}_{2}\right)^{p}$ for some Abelian group $\mathcal{A}$ of odd order, where $a=7 \cdot 2^{p-3}$.

Froncek [23] asked the following:

Problem 6.35. For what pairs $(n, r)$ there exists a handicap tournament $\operatorname{HIT}(n, r)$, or equivalently, an r-regular handicap distance antimagic graph with $n$ vertices?

Similarly, Froncek [25] asked the following:

Problem 6.36. For which triples $(n, g, d)$ there exists a g-regular handicap distance $d$-antimagic graph on $n$ vertices?

Observation 3.23 partially motivated Anholcer et al. to ask the following in [1].

Problem 6.37. Let $n$ and $p_{1}, \ldots, p_{m}$ be positive integers such that $p_{1}+\cdots+p_{m}=n$. When is it possible to find a partition of the set $\{1, \ldots, n\}$ into $m$ sets $A_{1}, \ldots, A_{m}$ such that $\left|A_{i}\right|=p_{i}$ and $\sum_{x \in A_{i}} x=\frac{\left(\begin{array}{c}n+1 \\ 2\end{array}\right)}{m}$ for every $i \in\{1, \ldots, m\}$.

If the above partition exists, then $n \equiv x(\bmod 2 m)$ where $x \in\{0,-1\}$.

Observation 6.38. [1] Assume that $p_{1}, \ldots, p_{m}$ are given in non-decreasing order. Let $P_{j}=\sum_{i=1}^{j} p_{i}$. If the mentioned partition exists, then for any $1 \leq j \leq m$, $\sum_{i=n-P_{j}+1}^{n} i \geq j \frac{\left(\begin{array}{c}n+1 \\ 2\end{array}\right)}{m}$. 


\section{Bibliography}

[1] M. Anholcer, S. Cichacz, and I. Peterin, Spectra of graphs and closed distance magic labelings, preprint (2014).

[2] B.D. Acharya, S.B. Rao, T. Singh, and V. Parameswaran, Neighborhood Magic Graphs, In National Conference on Graph Theory, Combinatorics, and Algorithms, (2004).

[3] M. Anholcer, S. Cichacz, I. Peterin and A. Tepeh, Group distance magic labeling of direct product of graphs, Ars Mathematica Contemporanea 9 (2015), 93-107.

[4] M. Anholcer, S. Cichacz, Note on distance magic products $G \circ C_{4}$, Graphs and Combinatorics, (2014).

[5] M. Anholcer, S. Cichacz, A. Görlich, Note on distance magic graphs $K_{m, n}\left[C_{4}\right]$, preprint.

[6] M. Anholcer, S. Cichacz, I. Peterin, A. Tepeh, Distance magic labeling and two products of graphs, arXiv:1210.1836 [math.CO].

[7] S. Arumugam, D. Froncek and N. Kamatchi, Distance magic graphs- a survey, J. Indones. Math. Soc., Special Edition (2011): 11-26.

[8] S. Arumugam, N. Kamatchi, and P. Kovář, Distance Magic Graphs, manuscript.

[9] S. Beena, On $\Sigma$ and $\Sigma^{\prime}$ labelled graphs, Discrete Mathematics, 309 (2009), 17831787.

[10] N. Biggs, Algebraic Graph Theory, Cambridge, (1974).

[11] J.A. Bondy and U.S.R. Murty, Graph Theory, Springer, 2008.

[12] S. Cichacz, Distance magic graphs $G \times C_{n}$, Preprint arXiv:1302.6561 (2013).

[13] S. Cichacz, Distance magic (r,t)-hypercycles, Utilitas Mathematica (2013), accepted.

[14] S. Cichacz, Group distance magic labeling of some cycle-related graphs, Australasian Journal of Combinatorics, 57, (2013), 235-243. 
[15] S. Cichacz, Note on group distance magic complete bipartite graphs, Central European Journal of Mathematics 12(3) (2014), 529-533.

[16] S. Cichacz, Note on Group Distance Magic Graphs $G\left[C_{4}\right]$, Graphs and Combinatorics, (2013).

[17] S. Cichacz and D. Froncek, Distance magic circulant graphs, Preprint Nr MD 071 (2013).

[18] S. Cichacz, D. Froncek, P.Gregor, P. Kovar, Distance magic hypercubes, manuscript.

[19] S. Cichacz, D. Froncek, K.A. Sugeng, and Sanming Zhou, Group distance magic and antimagic graphs, submitted, (2013).

[20] S. Cichacz and A. Gorlich, Constant sum partition of sets of integers and distance magic graphs, preprint.

[21] D. Froncek, Fair incomplete tournaments with odd number of teams and large number of games, Congr. Numer., 187 (2007), 83-89.

[22] D. Froncek, Group distance magic labeling of Cartesian products of cycles, Australasian Journal of Combinatorics, 55 (2013), 167-174.

[23] D. Froncek, Handicap distance antimagic graphs and incomplete tournaments, International Journal of Graphs and Combinatorics, 10 (2013), 199-127.

[24] D. Froncek, Incomplete round-robin tournaments and distance magic labelings, Abstracts of ICRTGC-2010.

[25] D. Froncek, Incomplete tournaments and handicap distance antimagic graphs, preprint.

[26] D. Froncek, P. Kovar, and T. Kovarova, Constructing distance magic graphs from regular graphs, J. Combin. Math. Comput., 78 (2011), 349-354.

[27] D. Froncek, P. Kovar, and T. Kovarova, Fair incomplete tournaments, Bulletin of the ICA, 48 (2006), 31-33.

[28] J. Gallian, A dynamic survey of graph labeling, Elec. J. Combin. DS6. http://www.combinatorics.org/Surveys/.

[29] C. Gavoille, D. Peleg, S. Pérennes and R. Raz, Distance labeling in graphs, Journal of Algorithms, 53 (2004), 85-112.

[30] P. Gregor and P. Kovar, Distance magic labelings of hypercubes, Electronic Notes in Discrete Mathematics, 40 (2013), 145-149. 
[31] J.R. Griggs and R.K. Yeh, Labelling graphs with a condition at distance 2, SIAM J. Discrete Math., 5(4), (1992), 586-595.

[32] R. Hammack, W. Imrich, and S. Klavžar, Handbook of Product Graphs, Second Edition, CRC Press, Boca Raton, FL, 2011.

[33] T. Harmuth, Ueber magische Quadrate und ähnliche Zahlenfiguren, Arch. Math. Phys., 66 (1881), 286-313.

[34] T. Harmuth, Ueber magische Rechtecke mit ungeraden Seitenzahlen, Arch. Math. Phys., 66 (1881), 413-447.

[35] M. Jinnah, On $\Sigma$-labelled graphs, In Technical Proceedings of Group Discussion on Graph Labeling Problems eds. B.D. Acharya and S.M. Hedge, (1999), 71-77.

[36] G.Y. Katona, H.A. Kierstead, Hamiltonian chains in hypergraphs, J. Graph Theory, 30, (1999), 205-212.

[37] P. Kovar, D. Froncek, and T. Kovarova, A note on 4-regular distance magic graphs, Australasian Journal of Combinatorics 54 (2012), 127-132.

[38] P. Kovar and A. Silber, Distance magic graphs of high regularity, Int. J. Graphs Comb., 9 (2012), 213-219.

[39] P. Kovar, A. Silber, P. Kabelikova, and M. Kravcenko, On regular distance magic graphs of odd order, preprint (2012).

[40] M. Miller, C. Rodger and R. Simanjuntak, Distance magic labelings of graphs, Australasian Journal of Combinatorics, 28 (2003), 305-315.

[41] A. O'Neal and P. Slater, An introduction to distance D magic graphs, J. Indonesian Math. Soc. Special Edition (2011), 89-107.

[42] A. O'Neal and P. Slater, An introduction to closed/open neighborhood sums: minimax, maximin, and spread, Mathematics in Computer Science, 5 (2011), 69-80.

[43] A. O'Neal and P. Slater, The minimax, maximin and spread values for open neighborhood sums for 2-regular graphs, Congr. Num. 208, (2011), 19-32.

[44] A. O'Neal and P. Slater, Uniqueness of vertex magic constants, SIAM J. Discrete Math, 27 (2013), 708-716.

[45] C. Pickover, The Math Book, Sterling, New York, (2009).

[46] S.B. Rao, Personal communication to S. Arumugam, (2010), published in [7]. 
[47] S.B. Rao, Sigma graphs-a survey, In Labelings of Discrete Structures and Applications, eds. B.D. Acharya, S. Arumugam, and A. Rosa, Narosa Publishing House, New Delhi, (2008), 135-140.

[48] S.B. Rao, T. Singh, and V. Parameswaran, Some sigma labelled graphs I, In Graphs Combinatorics, Algorithms and Applications, eds. S. Arumugam, B.D. Acharya, and S.B. Rao, Narosa Publishing House, New Delhi, (2004), 125-133.

[49] W.R. Scott. Group Theory. Prentice-Hall, Englewood Cliffs, N.J., 1964.

[50] J. Sedláček, Problem 27 in Theory of Graphs and its Applications, Proc. Symposium Smolenice 1963, Prague (1964), 163-164.

[51] M. Seoud, A. Abdel Maqsoud, and Y. Aldiban, New classes of graphs with and without 1-vertex magic vertex labeling, Proc. Pakistan Acad. Sci., 46 (2009), $159-174$.

[52] M.K. Shafiq, Ali G., and R. Simanjuntak, Distance magic labelings of a union of graphs, AKCE J. Graphs. Combin., 6(1) (2009), 191-200.

[53] R. Simanjuntak, M. Elviyenti, M.N. Jauhari, A.S. Praja, and I.A. Purwasih, Magic labelings of distance at most 2, 37th Australasian Conference on Combinatorial Mathematics and Combinatorial Computing, arXiv:1312.7633 [math.CO].

[54] K.A. Sugeng, D. Froncek, M. Miller, J. Ryan, and J. Walker, On distance magic labeling of graphs, J. Combin. Math. Combin. Comput., 71 (2009), 39-48.

[55] V. Vilfred, $\Sigma$-labelled graphs and Circulant Graphs, Ph.D. Thesis, University of Kerala, Trivandrum, India, (1994). 


\section{Index}

1-vertex-magic vertex labelings, 11

balanced, 48

balanced distance magic graph, 33

balanced labeling

$\Gamma$-balanced labeling, 57

$\mathbb{Z}_{n}$-balanced labeling, 46

balanced matrix, 49

bowtie product, 41

Cartesian product, 25, 53

Cayley graph, 47

chromatic number, 30

circulant, 17

complement, 23

complete bipartite graph, 9

complete graph, 15

connection set, 47

cycle, 15

degree, 13

diameter, 38

direct product, 24, 50, 56

distance, 10

distance $d$-antimagic labeling, 41

distance antimagic labeling

$\Gamma$-distance antimagic labeling, 54

distance magic, 11

closed distance magic, 34

D-distance magic, 38

distance magic hypergraph, 18

distance magic labeling, 11

$\Gamma$-distance magic labeling, 45

$D$-distance magic, 37

Dutch windmill graph, 23

edges, 9 embedding, 30

equalized incomplete tournament, 61

Eulerian cycle, 30

Eulerian graph, 30

exponent, 47

fair incomplete tournament, 61

fractional dominating function, 41

fractional domination number, 41

friendship graph, 23

graph, 9

group distance magic labeling, 45

Hamming graph, 53

handicap incomplete tournament, 62

hypercube, 47

$(\mathrm{r}, \mathrm{t})$-hypercycle, 18

hypergraph, 18

incidence function, 9

independent, 66

induced subgraph, 30

involution, 53

join graph, 28

lexicographic product, 22, 49

lifted magic rectangle, 31

line graph, 37

magic constant, $11,34,45$

$D$-distance magic constant, 37

magic labeling, 9

magic rectangle, 19

magic rectangle set, 43

magic square, 9

$t$-matching, 14 
multipartite graph, 19

neighbor-balanced, 48

open neighborhood, 11

order, 13

ordered distance antimagic graph, 42

ordered distance antimagic labeling, 42

parts, 19

path, 15

perfect code, 37

perfect matching, 15

power, 18

regular, 10

$r$-regular, 16

rounds, 61

set subtraction, 38

sigma labelings, 11

$\Sigma^{\prime}$-graphs, 34

size, 48

spectrum, 37

splitting graph, 29

strength, 61

strong product, 26, 58

strongly regular, 35

symmetric difference, 16

total strength of opponents, 61

tree, 15

trivial $D$-distance magic labeling, 39

twin vertices, 33

twins, 33

t-uniform, 18

vertices, 9

weight, 11, 45

wheel, 15 